\title{
Coulomb explosion imaging for gas-phase molecular structure determination: An $a b$ initio trajectory simulation study
}

Cite as: J. Chem. Phys. 153, 184201 (2020); https://doi.org/10.1063/5.0024833

Submitted: 11 August 2020 . Accepted: 25 October 2020 . Published Online: 12 November 2020

(D) Weiwei Zhou, (D) Lingfeng Ge (葛聆沨), (D) Graham A. Cooper, (D) Stuart W. Crane, Michael H. Evans, (D) Michael N. R. Ashfold, and (iD) Claire Vallance
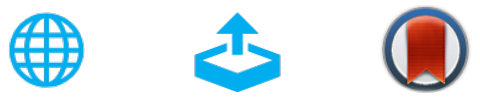

View Online

Export Citation

\section{ARTICLES YOU MAY BE INTERESTED IN}

Excited-state dynamics of $\mathrm{CH}_{2} \mathrm{I}_{2}$ and $\mathrm{CH}_{2} \mid \mathrm{Br}$ studied with UV-pump VUV-probe momentum-resolved photoion spectroscopy

The Journal of Chemical Physics 153, 184304 (2020); https://doi.org/10.1063/5.0026177

Coulomb explosion imaging of $\mathrm{CH}_{3} \mathrm{I}$ and $\mathrm{CH}_{2} \mathrm{Cll}$ photodissociation dynamics

The Journal of Chemical Physics 149, 204313 (2018); https://doi.org/10.1063/1.5041381

Velocity map imaging of ions and electrons using electrostatic lenses: Application in photoelectron and photofragment ion imaging of molecular oxygen

Review of Scientific Instruments 68, 3477 (1997); https://doi.org/10.1063/1.1148310

Challenge us.

What are your needs for periodic signal detection?

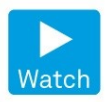

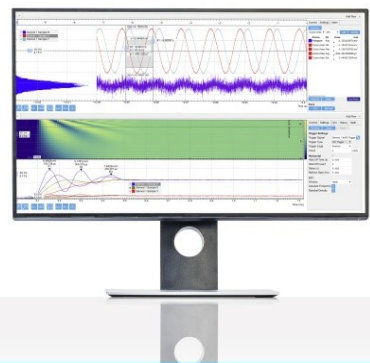

Zurich

Instruments 


\title{
Coulomb explosion imaging for gas-phase molecular structure determination: An ab initio trajectory simulation study
}

\author{
Cite as: J. Chem. Phys. 153, 184201 (2020); doi: 10.1063/5.0024833 \\ Submitted: 11 August 2020 - Accepted: 25 October 2020 • \\ Published Online: 12 November 2020
}

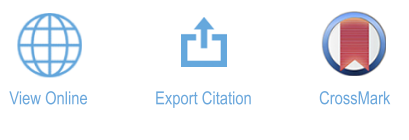

Weiwei Zhou, ${ }^{1, a)}$ (D) Lingfeng Ge (葛聆沨), ${ }^{2}$ (D) Graham A. Cooper, ${ }^{2, b)}$ (D) Stuart W. Crane, ${ }^{2}$ (D) Michael H. Evans, ${ }^{2}$ Michael N. R. Ashfold, ${ }^{2}$ (D) and Claire Vallance ${ }^{1, c)}$ (D)

\author{
AFFILIATIONS \\ 'Department of Chemistry, University of Oxford, Chemistry Research Laboratory, 12 Mansfield Rd., \\ Oxford OX1 3TA, United Kingdom \\ ${ }^{2}$ School of Chemistry, University of Bristol, Cantock's Close, Bristol BS8 1TS, United Kingdom
}

a) Present address: National Centre of Excellence in Mass Spectrometry Imaging (NiCE-MSI), National Physical Laboratory, Teddington TW11 OLW, United Kingdom.

b) Present address: Department of Chemistry, University of Missouri, 601 S College Ave., Columbia, MO 65211, USA.

${ }^{c}$ Author to whom correspondence should be addressed: claire.vallance@chem.ox.ac.uk

\begin{abstract}
Coulomb explosion velocity-map imaging is a new and potentially universal probe for gas-phase chemical dynamics studies, capable of yielding direct information on (time-evolving) molecular structure. The approach relies on a detailed understanding of the mapping between the initial atomic positions within the molecular structure of interest and the final velocities of the fragments formed via Coulomb explosion. Comprehensive on-the-fly ab initio trajectory studies of the Coulomb explosion dynamics are presented for two prototypical small molecules, formyl chloride and cis-1,2-dichloroethene, in order to explore conditions under which reliable structural information can be extracted from fragment velocity-map images. It is shown that for low parent ion charge states, the mapping from initial atomic positions to final fragment velocities is complex and very sensitive to the parent ion charge state as well as many other experimental and simulation parameters. For highcharge states, however, the mapping is much more straightforward and dominated by Coulombic interactions (moderated, if appropriate, by the requirements of overall spin conservation). This study proposes minimum requirements for the high-charge regime, highlights the need to work in this regime in order to obtain robust structural information from fragment velocity-map images, and suggests how quantitative structural information may be extracted from experimental data.
\end{abstract}

Published under license by AIP Publishing. https://doi.org/10.1063/5.0024833

\section{INTRODUCTION}

Several new and exciting experimental techniques for probing isolated (i.e., gas phase) molecules with high spatial and temporal resolution have recently been developed and are now sufficiently mature to provide fundamental insights into phenomena such as the time evolving coupled electron and nuclear dynamics of molecules immediately following photoexcitation. Examples include timeresolved photoelectron spectroscopy, ${ }^{1-5} \mathrm{x}$-ray scattering, ${ }^{6}$ electron diffraction, ${ }^{7-9}$ and transient $\mathrm{x}$-ray absorption spectroscopies. ${ }^{10-12}$ The key to each of these developments have been advances in the availability and the ease of use of ultrafast laser sources delivering femtosecond ( $\mathrm{fs}$ ) and sub-fs ${ }^{13}$ pulses across broad regions of the electromagnetic spectrum. These techniques all come with attendant challenges, however. Scattering and absorption methods require relatively high sample number or column densities, which limits the range of gas-phase systems amenable to study. Time-resolved photoelectron spectroscopy is much more sensitive: each molecule that is photoionized reports directly via the ejected electron. However, as with each of these new techniques, any full analysis of the measured data is heavily dependent on the availability of similar cutting-edge theory, e.g., electronic structure calculations for the states involved 
in both the pump and the probe steps, and proper treatment of the excited state dynamics encompassing any non-adiabatic couplings en route to the ultimate products.

Coulomb explosion imaging (CEI) - and its more recent variant Coulomb explosion velocity-map imaging (CE-VMI) - is another emerging technique capable of mapping photoinduced unimolecular reactions with high spatial and temporal resolution. ${ }^{14-40}$ A Coulomb explosion (CE) is a type of dissociation that occurs in highly charged ions formed by rapid stripping of a large number of valence electrons from a molecule. Removal of valence electrons significantly disrupts, or even destroys, the chemical bonding, resulting in a set of proximal positively charged atomic or molecular fragments. The Coulomb repulsion between these fragments leads to rapid dissociation, or "explosion," on a fs timescale. Given two assumptions, namely, (i) the initial ionization event is sufficiently fast that the nuclei do not move significantly while the electrons are being stripped and (ii) the CE is "complete" (i.e., the molecule dissociates into atomic ions), the initial atomic positions should be mapped in a well-defined way onto the final atomic fragment velocities during the $\mathrm{CE}$. Measurement of these final atomic velocities can thus, in principle at least, provide detailed information on both the dissociation dynamics and the starting molecular structure.

Various approaches have been employed to initiate CEs experimentally. In early experiments, beams of singly charged ions were accelerated to extremely high kinetic energies and passed through a thin metal "stripping foil" to remove valence electrons. ${ }^{41}$ These pioneering experiments, and follow-up simulations, illustrated very clearly the potential of CEI methods for determining the structure (stereochemistry) of then very topical small polyatomic cations such as $\mathrm{C}_{2} \mathrm{H}_{3}{ }^{+42-44}$ and $\mathrm{CH}_{4}{ }^{+}{ }^{45}$ In more recent approaches, the molecule of interest is prepared in a molecular beam, which is intersected by an ultrafast laser pulse, as shown schematically for a CE-VMI experiment in Fig. 1. Interaction with a high-intensity infrared fs laser pulse, for example, can lead to loss of multiple valence electrons-via multiphoton ionization or strong-field ionization, or some combination of the two. ${ }^{31}$ If, as is usually the case, the laser pulse is linearly polarized, it results in an aligned sample of highly charged parent cations primed for explosion. In some experiments, an additional longer-duration strong-field "prepulse" is used to align or orient the neutral parent ions prior to interaction with the fs pulse that initiates the CE. ${ }^{22-24,26}$ As an alternative to a high-intensity infrared pulse, an extreme ultraviolet (XUV) laser pulse can be used to ionize one or more core electrons, with multiple valence ionization and subsequent CE occurring following an Auger cascade. The nuclear dynamics of the CE appear to be relatively insensitive to the mechanism of the initial ionization event, though comparative studies have suggested that ultrafast XUV photoexcitation may result in somewhat faster ionization and fragmentation. ${ }^{2}$

In the setup shown in Fig. 1, the nascent CE products are accelerated by an electric field along a flight tube to a position-sensitive detector. Fragment ions with different mass-to-charge $(\mathrm{m} / \mathrm{z})$ ratios are accelerated to different extents by the extraction field and become separated in time as they traverse the flight tube. The ion detector typically comprises a pair of microchannel plates coupled to a fast phosphor screen, which generates a scintillation event for each incident ion. The extraction field is usually tuned such that the scintillation events recorded at a particular arrival time constitute a two-dimensional (2D) projection of the full three-dimensional (3D) scattering or velocity distribution of the corresponding fragment ion. ${ }^{46}$ Coupling the phosphor screen to an ultra fast event-triggered pixel sensor such as the PImMS ${ }^{47,48}$ or TimePix ${ }^{49}$ sensor allows an $(x, y, t)$ data point to be recorded and stored for each detected ion. The resulting dataset can be integrated over the position $(x, y)$ to yield a time-of-flight (TOF) mass spectrum of the CE products, enabling their identification and quantification, or can be integrated over the relevant time interval to obtain the spatial distribution for a given product ion. More recently, the application of covariance mapping to such datasets has enabled extraction of correlated velocity distributions of two or more ions, allowing the relative velocities of different product pairs or triplets to be explored. ${ }^{26,40}$ In the case of CE products, this provides direct structural information on the parent molecule via the mapping from the initial atomic position to the final atomic ion velocity.

While still in its infancy, the technique holds promise both as a method for gas-phase structure determination and when used as the probe step in an ultrafast pump-probe experiment, for following dynamics in real time and recording "molecular movies" of chemical processes. CE-VMI has already been used to obtain structural information on, for example, a halogenated biphenyl molecule in its ground state $^{23,24}$ and following torsional vibrational excitation, ${ }^{22}$ to

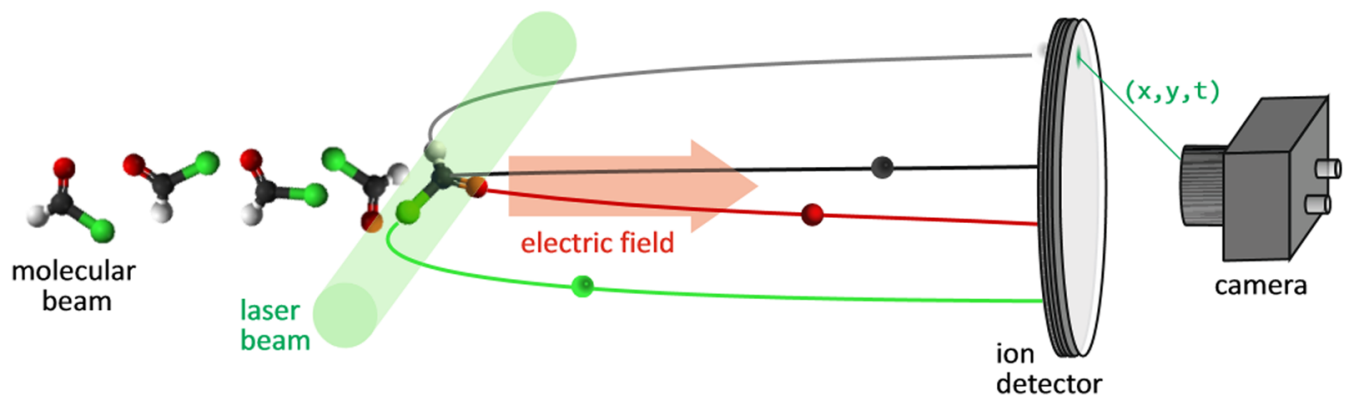

FIG. 1. Schematic of a Coulomb explosion velocity-map imaging experiment. A molecular beam is crossed with a high-intensity ultrafast laser pulse, which initiates the Coulomb explosion. During the CE, the initial positions of the atoms within the molecule are mapped onto their final velocities. The charged atomic and/or molecular fragments are accelerated toward a position-sensitive detector by an electric field, yielding an $(x, y, t)$ data point for each fragment ion. The ion arrival times correlate with their mass-to-charge $(\mathrm{m} / \mathrm{z})$ ratios, and the arrival positions correlate with their velocities. 
distinguish between different structural isomers of difluoroiodobenzene $^{32}$ and to determine the absolute configurations of the foregoing biphenyl molecule ${ }^{26}$ and of $\mathrm{CHFClBr}{ }^{2}$

The ability to extract structural information from velocity-map images of CE products depends critically on the extent to which the mapping between the initial position and the final fragment velocity is understood. Most previous simulations of CE processes have been based on a simple model in which the Coulomb repulsion between the charged fragments is the only force acting. ${ }^{50-52}$ As shown here, this is likely to be a reasonable approximation for very highly charged parent ions but is less likely to be valid for parent ions prepared in lower charge states. A limited number of studies have been performed in which reduced-dimensionality $a b$ initio potential energy surfaces have been used to investigate breaking of a single bond via a CE mechanism. ${ }^{18,35,53}$ The effect of the laser field and ionelectron interactions on the $\mathrm{CE}$ of benzene and hexafluorobenzene has been studied experimentally and by molecular dynamics (MD) simulations, ${ }^{54,55}$ and Wang et al. ${ }^{56}$ used the time-dependent local density approximation coupled with MD methods to investigate the $\mathrm{CE}$ (and two other dissociation mechanisms) of water dimers. However, we are not aware of any comprehensive $a b$ initio trajectory simulations of CEs for polyatomic molecules in which all chemical binding forces and all degrees of freedom have been considered in parallel with the Coulomb repulsion.

Here, we present the results of on-the-fly $a b$ initio trajectory simulations of the CE of two prototypical small planar molecules, formyl chloride and cis-1,2-dichloroethene, chosen to illustrate electronic similarities (replacing oxygen in the former by chlorocarbene yields the latter) but also obvious symmetry-related differences. Different $a b$ initio electronic structure methods have been tested, and calculations are performed to determine the forces acting on each atom at each trajectory time step, ensuring that all relevant chemical forces are considered throughout the trajectory. The approach reveals many of the key factors affecting the products of a $\mathrm{CE}$, their velocities, and how these are influenced by the initial parent ionization.

\section{METHODS}

Simulation strategies were developed independently in Oxford and in Bristol. The respective approaches have much in common but differ in some details, as described below. Section III presents data from the various simulations.

The software developed in Oxford to perform the CE trajectory simulations was coded into a single executable MATLAB program (both Bash and Python scripts were used in Bristol), with calls to the Gaussian electronic structure package ${ }^{58}$ at each time step to perform the electronic structure calculations required to determine the forces on each atom. In all cases, the following steps were performed during each simulation:

1. The geometry of the neutral molecule was optimized using the electronic structure method of choice (details of these calculations appear later). The molecular geometry is assumed not to change during the ionization step, unless specified otherwise, so that the chosen geometry of the neutral molecule defines the initial geometry of the ion.
2. A single-point energy calculation was performed [at the unrestricted Hartree-Fock (UHF) level] at this geometry for the parent ion with the user-defined charge $Z+$ (using the converged wavefunction from the previous cycle on the second and subsequent time steps). Throughout, upper case $Z$ will be used to define the number of positive charges on the parent ion of interest, and lower case $z$ to indicate the fragment ion charges. The stability of the wavefunction at each subsequent time step was tested within Gaussian and re-optimized (at the same level of theory) if an instability was found. ${ }^{59,60}$ The resulting wavefunction was then saved to a checkpoint file.

3. Using the wavefunction from step 2, a single-point calculation was performed at each time step to determine the forces on each atom as well as the atomic partial charges according to the Merz-Singh-Kollman ${ }^{61,62}$ (in Oxford only), Natural Bond Order (NBO), ${ }^{63}$ and Hirshfeld ${ }^{64}$ population analyses. The criterion for basis set linear dependence in the NBO analysis was set at $10^{-8}$ (Oxford) and $10^{-7}$ (Bristol), respectively (cf. the default value is $10^{-6}$ ). The NBO population analysis also returns the Wiberg bond index (WBI), ${ }^{65}$ a measure of the bond order between any two atoms.

4. Using the starting geometry obtained in step 1 and the forces obtained in step 3, and assuming all initial atomic velocities to be zero, Newton's equations of motion were integrated using, for example, the ode45 ordinary differential equation (ODE) solver within MATLAB (Oxford), i.e.,

$$
\boldsymbol{F}_{i}=m_{i} \sum_{j} \boldsymbol{a}_{i j}=m_{i} \sum_{j} \frac{d^{2} \boldsymbol{r}_{i j}}{d t^{2}},
$$

where $\boldsymbol{F}_{i}, m_{\mathrm{i}}, \boldsymbol{a}_{i j}$, and $\boldsymbol{r}_{i j}$ are the force acting on the atom $i$, the mass of the atom $i$, the acceleration of the atom $i$ due to the presence of the atom $j$, and the distance between atoms $i$ and $j$, respectively. The ode 45 solver chosen for the simulations is a general purpose ODE solver for non-stiff problems, ${ }^{66}$ which uses an explicit Runge-Kutta algorithm employing the Dormand-Prince $(4,5)$ pair. ${ }^{67}$ The size of the integration time step is varied automatically over the course of the trajectory according to the force (i.e., the gradient of the potential energy returned by the electronic structure calculation at each time step) in order to achieve the best compromise between run time and accuracy of the integration. The resulting time steps in the first part of the simulation when the forces are changing rapidly are on the order $10^{-3} \mathrm{fs}-10^{-1} \mathrm{fs}$. The Bristol approaches were very similar. Both use the initial velocity from the previous step, the acceleration (determined from the calculated forces), and the time until the next time step as inputs for Newton's equations of motion to predict the distance traveled in the proceeding step and the final velocity achieved, before passing these outputs to the next iteration of the loop. The nuclear positions in the Bash calculations were updated in $0.1 \mathrm{fs}$ intervals, whereas the integration time step in the Python calculations was varied according to the interatomic separations. Again, the starting time step was $0.1 \mathrm{fs}$ until the distances between all atom pairs exceeded the sum of their respective van der Waals radii. Once this criterion was met, the calculation stepped to the next integer number of fs and proceeded in $1 \mathrm{fs}$ intervals until the minimum distance 
between atoms was $>7 \AA$. After surpassing this distance, the time step was increased to the next multiple of 10 and continued in $10 \mathrm{fs}$ intervals. If at any point the distances between any pair of fragments decreased so that one of the foregoing criteria was no longer fulfilled, the program continued from that point at the shorter time step. Again, the trajectory time point, atomic partial charges, velocities, coordinates, and forces as well as the total energy of the system were all saved for later analysis.

5. Steps 2 through 4 were then iterated using the output geometry from step 4 as the new input geometry for the next cycle. The calculations were terminated once some user-defined criterion had been satisfied, e.g., the total trajectory time, all atomic forces falling below a pre-defined threshold, or atomic velocities changing by less than a pre-defined amount at each step.

Extensive comparisons were made in both Oxford and Bristol, using various test molecules, to determine the level of theory and basis set that provided an acceptable performance according to a series of convergence tests while still allowing the calculations to run in a reasonable time. Theoretical models tested in Oxford included Hartree-Fock (HF) theory, ${ }^{68,69}$ second to fourth order Møller-Plesset perturbation theory (MP2, MP3, MP4), ${ }^{70-78}$ quadratic configuration interaction (QCISD), and density functional theory (DFT) employing the B3LYP functional. ${ }^{79-82} \mathrm{HF}$ and MP2 methods and further functionals (including CAM-B3LYP, $\omega B 97, \omega B 97 x,{ }^{84,85}$ LC- $\omega$ PBE, ${ }^{86-88}$ LC-BLYP, ${ }^{79,80,89,90} \mathrm{M} 11,{ }^{91}$ and M06- $\mathrm{HF}^{92}$ ) were investigated in Bristol. Only the HF, MP2, and DFT methods were found to be sufficiently fast to be realistic options for further simulations with the available resources. For most molecules tested, DFT methods often returned physically unreasonable noninteger charges on the final dissociation products. Unrestricted (U) MP2 and HF methods, in contrast, both consistently returned integer charges. UMP2 was adopted as the method of choice for most of the studies reported here and, in several instances, the outcomes compared with those predicted using UHF methods.

Several different basis sets were tested to investigate the effects of the number of contracted Gaussians used to describe the valence orbitals and the inclusion of polarization and diffuse functions. The 6-31G(d,p), ${ }^{93-97}$ 6-311G(d,p), 6-311G(2d,p), 6-311 + G(d,p), ${ }^{98-101}$ and cc-pVTZ basis sets ${ }^{102-108}$ were each investigated in Oxford. ${ }^{56}$ The fact that valence polarization and diffuse functions were found to have little effect is not that surprising, given that many of the valence electrons have been removed in the highly charged ionic systems under study. The 6-311G(d,p) basis set was chosen in Oxford to provide a good compromise between computational accuracy and run time for the small molecules of current interest, while the Bristol calculations employed the aug-cc-pVDZ (which was found to provide adequate performance, comparable with the triple-zeta equivalent) or def2-TZVP basis sets. ${ }^{104-107}$

For most simulations, the parent ion was assumed to be vibrationless. In any real application of CEI methods, however, the ensemble of parent molecules will span a range of geometries and initial nuclear momenta. In the simplest case, this span will be determined by the amplitudes of the zero-point motions, but more extensive nuclear motions would need to be considered when CEI methods are used in ultrafast time-resolved measurements of dissociating or isomerizing parent molecules. Here, we choose simply to illustrate some of the potential effects of zero-point vibrational motion. The force constants and reduced masses determined from the electronic structure calculations for each (harmonic) normal mode of vibration were used to calculate the classical turning points. The normal coordinate vectors returned by the electronic structure calculations were then used to determine the maximum atomic displacements from the equilibrium geometry due to zero-point energy in each normal mode. CE simulations were then started from these maximally distorted geometries.

Further variables considered in this study were the electronic state of the parent cation and the timescale of its preparation. The Oxford simulations were limited to the lowest energy singlet/doublet spin state (for even/odd charge $Z+$, respectively), but each $Z+$ ion will support many different electronic states and there is no a priori reason why strong-field ionization should produce any given $Z+$ ion solely in its ground electronic state (nor that the ground state should have singlet/doublet spin multiplicity). Electronic excitation effects have not been investigated here in any detail, but the Bristol calculations explored a greater range of spin multiplicities, identifying both the ground state and the lowest energy state (or states) of any given $Z+$ ion with a spin multiplicity different from that of the ground state, and, for several $Z+$ charge states of both formyl chloride and cis-1,2-dichloroethene, explored the sensitivity of the ensuing CE dynamics to the choice of the spin state.

Theoretical treatments of the electron dynamics in the $\mathrm{CE}$ range from the charge resonance-enhanced ionization model ${ }^{109}-$ which assumes some initial ionization that drives nuclear distortion, facilitating further excitation and ionization leading to the CEto the ionization ignition model, ${ }^{110}$ wherein the parent cation is assumed to be formed instantaneously relative to the timescale of nuclear motion. Most of the model results reported here assume direct vertical ionization to the parent ion charge state of interest, but a limited number of calculations have also been run to explore the extent to which the CE dynamics depend on the pathway by which the polycation is formed.

\section{RESULTS AND DISCUSSION}

We report the results of calculations performed on formyl chloride and cis-1,2-dichloroethene (henceforth often abbreviated as FC and c-DCE, respectively) using one of the five combinations of the electronic structure method, basis set, and trajectory step size, unless specifically stated otherwise. The five combinations, labeled I, IIa, IIb, IIIa, and IIIb, are defined in Table I.

During the early exploratory part of this study, more limited sets of simulations (not presented here) were also performed for several other small organic molecules, including trans-2,3dideuterooxirane, chloroiodomethane, and fluoromethane, using method I.

Numerous simulations for the molecules of present interest were performed for $Z+$ charge states ranging from $Z=2$ up to the value corresponding to complete removal of all valence electrons (i.e., $Z=18$ in the case of FC, $Z=24$ for c-DCE). Those reported in the main text have been chosen to address and demonstrate the key conclusions of the present work. Further simulations designed to explore further dynamical aspects and support the key conclusions are presented as the supplementary material. 
TABLE I. Summary of simulation methods employed.

\begin{tabular}{lccc}
\hline \hline Method & $\begin{array}{c}\text { Electronic } \\
\text { structure method }\end{array}$ & Basis set & Trajectory step size (s)/fs \\
\hline I & UMP2 & $6-311 \mathrm{G}(\mathrm{d}, \mathrm{p})$ & Determined by the ODE solver in MATLAB \\
IIa & UMP2 & aug-cc-pVDZ & $0.1 / 1 / 10$ (see Sec. II) \\
IIb & UHF & aug-cc-pVDZ & $0.1 / 1 / 10$ (see Sec. II) \\
IIIa & UMP2 & def2-TZVP & 0.1 \\
IIIb & UHF & def2-TZVP & 0.1 \\
\hline \hline
\end{tabular}

Both molecules have planar equilibrium geometries, which define the $x y$ plane in the simulations. As noted above, the time, atomic charges, interatomic forces, atomic positions, atomic velocities, WBIs between all pairs of atoms, and the total energy of the system were recorded at each trajectory time step, with the simulations running for total trajectory times of (up to) $500 \mathrm{fs}$. The method I calculations show better total energy conservation, a consequence of the greater number of shorter time steps used in the early stages of the calculation. However, like-for-like comparisons of the method I and IIa outputs revealed no differences in the identities of predicted products from the $\mathrm{CE}$ of any given parent cation, though electronic structure differences were noted in the products arising in the CE of the FC 4+ cation. Of greater importance in the current context, the various method outputs predicted minimal differences in the product velocities following the $\mathrm{CE}$ from the higher $Z+$ cations. Sample test data are shown in Sec. S1 (Figs. S1-S6) in the supplementary material.

Trajectories running for many different initial charge states identify different dissociation behaviors when in the "low" and "high" charge regimes. For the purposes of this discussion, "low" and "high" charge states are defined, respectively, as those that dissociate to yield a mixture of atomic and molecular fragments and those for which fragmentation results in direct and exclusive formation of atomic ions. The appropriate classification is generally obvious when inspecting the fragment ions (and their charges) resulting from the $\mathrm{CE}$ simulations. However, there are potential ambiguities in the case of low charge states, as fragment ions formed with sufficient internal excitation may further decompose on longer timescales. For consistency, therefore, the fragment ions arising from the CE of a range of initial charge states for both molecules listed in Tables II and III are taken to be those present at $t=50 \mathrm{fs}$. Fragments are classified as "molecular" at that time if the separations between any pair or pairs of constituent nuclei are $\leq 2.5 \AA$.

\section{A. Low parent ion charge states}

Tables II and III detail the atomic and molecular products predicted (at $t=50 \mathrm{fs}$ ) following the CE of FC ions with $Z=3-5$ and c-DCE ions with $Z=3-7$ in their respective ground electronic states and in the lowest energy states of other spin multiplicity. The energy difference between the different spin states of a given parent ion is small compared with the total ionization energy, $I E$, and-as shown in Table III-the predicted energetic ordering of these spin states can vary with the choice of the electronic structure method. So, too, can the details of the subsequent fragmentation due, in part at least, to small differences in the molecular geometries obtained using the different electronic structure methods that define the initial atomic positions for the CE simulation. In all cases, however, all primary products from the $\mathrm{CE}$ of the cations of both molecules are charged, i.e., no early-time neutral fragments are predicted from either molecule. Based on the above definition, the high-charge state regime for $\mathrm{FC}$ is found to begin at $Z=6$, while for c-DCE, the onset is at $Z=8$.

\section{Formyl chloride}

Figure 2 shows the fragment trajectories returned by method IIa following vertical excitation from the ground-state equilibrium geometry of FC to the ground states of its $3+, 4+$, and $5+$ cations, along with the evolving atomic charges, atomic speeds, and WBIs. The point $(0,0)$ defines the center of mass, and this figure also serves to define the color coding used throughout: $\mathrm{H}, \mathrm{C}, \mathrm{Cl}$, and $\mathrm{O}$ atoms are depicted in black/white, gray, green, and red, respectively, with black, green, and red also used for the respective bonds to the $\mathrm{C}$ atom (and gray for the $\mathrm{C}-\mathrm{C}$ bond in $\mathrm{c}-\mathrm{DCE}$ ).

These data serve to illustrate the diverse, $Z$-dependent fragmentation behavior of the low charge state parent cations. The groundstate $3+$ cation is predicted to start separating into $\mathrm{HCO}^{+}+\mathrm{Cl}^{2+}$ fragments, but subsequent charge transfer triggers further dissociation of the $\mathrm{HCO}$ moiety yielding $\mathrm{H}^{+}, \mathrm{CO}^{+}$, and $\mathrm{Cl}^{+}$as the asymptotic fragments. The light $\mathrm{H}^{+}$ions arising from the $\mathrm{CE}$ of the $4+$ and $5+$ cations are predicted to have much higher recoil velocities than the heavier partner fragments; such behavior is characteristic of all higher parent charge states also, as shown in Sec. III B. The middle column of Fig. 2 shows the $4+$ cation dissociating to $\mathrm{H}^{+}, \mathrm{CO}^{+}$, and $\mathrm{Cl}^{2+}$ fragments and illustrates the rotational and vibrational excitation of the $\mathrm{CO}^{+}$fragment. Data for the $4+$ cation calculated using methods IIa and I, compared in Fig. S3, suggest that the former method typically predicts excessive vibrational excitation of any molecular fragments. We suspect that this is another consequence of the longer time steps used in the short range region of the method IIa trajectory simulations. The data for the $4+$ cation also serve to highlight the continuing influence of valence bonding in these low charge states. Given a CO first ionization energy of $14.01 \mathrm{eV},{ }^{111}$ the thermochemical threshold for this predicted combination of products lies $\sim 12.7 \mathrm{eV}$ higher than that for forming four singly charged atomic ions. The $5+$ cation, in contrast, is predicted to yield $\mathrm{H}^{+}+\mathrm{C}^{+}+\mathrm{O}^{+}+\mathrm{Cl}^{2+}$ fragment ions, via a transitory $\mathrm{CCl}^{3+}$ molecular fragment. The calculations thus suggest that the 5+ state of FC lies at the boundary between our definitions of low and high parent ion charge states. From these various simulations, we conclude that for 
TABLE II. Summary of ionic product species predicted following the CE of formyl chloride from the lowest energy parent cations of differing spin multiplicity $(2 S+1)$ and charge $Z+$, in the range $3 \leq Z \leq 18$, with the vertical ionization energy (IE) calculated using method lla and analyzed at $t=50 \mathrm{fs}$. The ground-state energy in each case is highlighted in bold. Also shown for the high parent charge states are the number of positive charges $\left(z_{\mathrm{i}}\right)$ on the atomic cations $i\left(z_{\mathrm{H}}\right.$ is unity in all cases and thus not listed), the spin multiplicity of the atomic state $\left(2 s+1\right.$, indicated by the left superscript), and $\sum I E\left(i, z_{\mathrm{i}}\right)$, the lowest sum of ionization energies for forming the specified atomic ions. The latter values are taken from the NIST Atomic Database, and the threshold energies for the various $z_{i}+\rightarrow\left(z_{i}+1\right)+$ transitions and energies of the various excited state ions are listed in Tables $\mathrm{S} 1$ and $\mathrm{S} 2$. The predicted product charge states in rows containing no such entry are as for the parent $Z$ and $2 S+1$ state in the immediate preceding row. The missing entry for the product ion energy sum following the $\mathrm{CE}$ of the quintet $\mathrm{FC}^{16+}$ ion reflects the lack of an excitation energy for the lowest quartet state of the $\mathrm{O}^{6+}$ ion.

\begin{tabular}{|c|c|c|c|c|c|c|}
\hline$Z$ & $2 S+1$ & $I E(\mathrm{eV})$ & \multicolumn{4}{|c|}{ Product ions } \\
\hline \multirow[t]{2}{*}{3} & 2 & 60.4 & \multicolumn{4}{|c|}{$\mathrm{Cl}^{+}$and $\mathrm{CHO}^{2+}$} \\
\hline & 4 & 60.2 & \multicolumn{4}{|c|}{$\mathrm{Cl}^{2+}$ and $\mathrm{CHO}^{+}$} \\
\hline \multirow[t]{3}{*}{4} & 1 & 98.4 & \\
\hline & 3 & 98.8 & \multicolumn{4}{|c|}{$\mathrm{H}^{+}, \mathrm{O}^{+}$, and $\mathrm{CCl}^{2+}$} \\
\hline & 5 & 99.4 & \multicolumn{4}{|c|}{$\mathrm{H}^{+}, \mathrm{C}^{+}, \mathrm{O}^{+}$, and $\mathrm{Cl}^{+}$} \\
\hline \multirow[t]{3}{*}{5} & 2 & 147.6 & \multirow{2}{*}{\multicolumn{4}{|c|}{$\begin{array}{c}\mathrm{H}^{+}, \mathrm{O}^{+} \text {, and } \mathrm{CCl}^{3+} \\
\mathrm{H}^{+}, \mathrm{C}^{+}, \mathrm{O}^{+} \text {, and } \mathrm{Cl}^{2+}\end{array}$}} \\
\hline & 4 & 147.7 & & & & \\
\hline & & & ${ }^{2 s+1} z_{\mathrm{C}}$ & ${ }^{2 \mathrm{~s}+1} z_{\mathrm{O}}$ & ${ }^{2 s+1} z_{\mathrm{Cl}}$ & $\sum I E\left(i, z_{i}\right)(\mathrm{eV})$ \\
\hline \multirow[t]{3}{*}{6} & 1 & 197.9 & ${ }^{1} 2$ & ${ }^{4} 1$ & ${ }^{4} 2$ & 99.6 \\
\hline & 3 & 207.0 & ${ }^{1} 2$ & ${ }^{4} 1$ & ${ }^{2} 2$ & 101.8 \\
\hline & 5 & 207.1 & & & & \\
\hline \multirow[t]{2}{*}{7} & 2 & 275.7 & ${ }^{1} 2$ & ${ }^{4} 1$ & ${ }^{3} 3$ & 139.4 \\
\hline & 4 & 275.6 & ${ }^{1} 2$ & ${ }^{3} 2$ & ${ }^{2} 2$ & 137.0 \\
\hline \multirow[t]{3}{*}{8} & 1 & 357.1 & ${ }^{1} 2$ & ${ }^{3} 2$ & ${ }^{3} 3$ & 174.6 \\
\hline & 3 & 355.2 & & & & \\
\hline & 5 & 353.4 & & & & \\
\hline \multirow[t]{2}{*}{9} & 2 & 444.5 & ${ }^{2} 3$ & ${ }^{3} 2$ & ${ }^{3} 3$ & 222.5 \\
\hline & 4 & 442.7 & & & & \\
\hline \multirow[t]{3}{*}{10} & 1 & 546.7 & ${ }^{2} 3$ & ${ }^{3} 2$ & ${ }^{2} 4$ & 275.7 \\
\hline & 3 & 546.2 & & & & \\
\hline & 5 & 545.9 & & & & \\
\hline \multirow[t]{2}{*}{11} & 2 & 661.1 & ${ }^{2} 3$ & ${ }^{2} 3$ & ${ }^{2} 4$ & 330.6 \\
\hline & 4 & 659.2 & & & & \\
\hline \multirow[t]{3}{*}{12} & 1 & 787.7 & ${ }^{1} 4$ & ${ }^{2} 3$ & ${ }^{2} 4$ & 395.1 \\
\hline & 3 & 785.3 & & & & \\
\hline & 5 & 795.5 & ${ }^{1} 4$ & ${ }^{4} 3$ & ${ }^{2} 4$ & 404.0 \\
\hline \multirow[t]{2}{*}{13} & 2 & 923.8 & ${ }^{1} 4$ & ${ }^{2} 3$ & ${ }^{1} 5$ & 462.8 \\
\hline & 4 & 933.0 & ${ }^{1} 4$ & ${ }^{2} 3$ & ${ }^{3} 5$ & 475.0 \\
\hline \multirow[t]{3}{*}{14} & 1 & 1081.6 & ${ }^{1} 4$ & ${ }^{1} 4$ & ${ }^{1} 5$ & 540.2 \\
\hline & 3 & 1081.8 & ${ }^{1} 4$ & ${ }^{3} 4$ & ${ }^{1} 5$ & 550.4 \\
\hline & 5 & 1100.8 & ${ }^{1} 4$ & ${ }^{3} 4$ & ${ }^{3} 5$ & 562.6 \\
\hline \multirow[t]{2}{*}{15} & 2 & 1248.7 & ${ }^{1} 4$ & ${ }^{1} 4$ & ${ }^{2} 6$ & 637.2 \\
\hline & 4 & 1266.6 & ${ }^{1} 4$ & ${ }^{3} 4$ & ${ }^{2} 6$ & 647.4 \\
\hline \multirow[t]{3}{*}{16} & 1 & 1433.3 & ${ }^{1} 4$ & ${ }^{2} 5$ & ${ }^{2} 6$ & 751.1 \\
\hline & 3 & 1452.3 & & & & \\
\hline & 5 & 1655.7 & ${ }^{1} 4$ & ${ }^{2} 5$ & ${ }^{4} 6$ & \\
\hline
\end{tabular}


TABLE II. (continued.)

\begin{tabular}{lcccccr}
\hline \hline$Z$ & $2 S+1$ & $I E(\mathrm{eV})$ & \multicolumn{4}{c}{ Product ions } \\
\hline 17 & 2 & $\mathbf{1 6 4 9 . 9}$ & ${ }^{1} 4$ & ${ }^{2} 5$ & ${ }^{1} 7$ & 865.3 \\
& 4 & 1851.3 & ${ }^{1} 4$ & ${ }^{2} 5$ & ${ }^{3} 7$ & 1074.8 \\
18 & & 1895.9 & ${ }^{1} 4$ & ${ }^{1} 6$ & ${ }^{1} 7$ & 1003.4 \\
& 1 & 2064.6 & ${ }^{1} 4$ & ${ }^{2} 5$ & ${ }^{2} 8$ & 1213.6 \\
& 3 & 2298.2 & ${ }^{1} 4$ & ${ }^{2} 5$ & ${ }^{4} 8$ & 1447.8 \\
\hline \hline
\end{tabular}

low charge states, both the identities of the primary products of the $\mathrm{CE}$ and the charges on these products are heavily dependent on the value of $Z$.

Several further datasets for the 4+ cation of FC serve to highlight the sensitivity of the predicted fragmentation to assumptions about the geometry or the electronic state of the parent ion, and the choice of the theoretical method. For example, trajectories launched from geometries corresponding to the classical turning points of different zero-point vibrational motions of the neutral molecule always show the $\mathrm{H}^{+}$ion leaving in much the same direction, with a similar velocity, but the remaining three fragments can experience a range of fates. A single plot showing all these outcomes from all starting positions is incomprehensible, so Fig. S7 in Sec. S2 of the supplementary material simply provides illustrative data comparing the outcomes of CEs initiated following vertical excitation from the ground-state equilibrium geometry and from the inner and outer turning points of the zero-point $\mathrm{C}=\mathrm{O}$ stretching motion in the ground-state neutral molecule (all calculated by method IIa). The CE at the inner turning point of this motion is predicted to result in a similar fragmentation behavior to that found when starting from the groundstate equilibrium geometry, though with reduced vibrational excitation in the $\mathrm{CO}^{+}$product. The latter, in contrast, shows prompt $\mathrm{H}^{+}$loss and subsequent slower breakup of the $\mathrm{ClCO}^{3+}$ partner into three singly charged atomic ions. Further variations in fragmentation behavior-in terms of product ions and their trajectorieswere noted when starting from an electronic state other than the ground state of the cation. Figure S8, for example, suggests that the CE from the lowest triplet state of the $4+$ cation of FC (calculated by method IIa), which lies just $0.4 \mathrm{eV}$ above the ground (singlet) state, favors prompt loss of $\mathrm{H}^{+}$and $\mathrm{O}^{+}$ions, and formation of a transient $\mathrm{CCl}^{2+}$ species that subsequently decays to singly charged atoms.

Additional calculations for the ground state of the 4+ cation of FC shown in Fig. S9 illustrate the sensitivity of the outcome to the choice of the electronic structure method. B3LYP returns a broadly similar fragmentation behavior to that found using method IIa (or method I), though it predicts non-integer charges on the asymptotic $\mathrm{Cl}$ and $\mathrm{CO}$ fragments. HF calculations (method IIb) return a rather different fragmentation behavior, wherein initial $\mathrm{H}^{+}$loss is followed by slower breakup of the $\mathrm{ClCO}^{3+}$ partner into its constituent singly charged atomic ions-more reminiscent of that found (by MP2 methods) when ionizing from the outer turning point of the zero-point $\mathrm{C}=\mathrm{O}$ stretch vibration. Such a different predicted behavior between MP2 and HF methods is unsurprising: even a cursory comparison of the atomic charges in the parent ion and in the final ionic fragments reveals that considerable charge transfer must occur on very short timescales. Correct treatment of electron correlation is thus likely to be important, suggesting that HF methods should be used only with due caution (especially when attempting to model the CE of cations in low charge states).

\section{2. cis-1,2-dichloroethene}

Figure 3 compares the calculated (by method IIIa) fragment ion trajectories following vertical excitation from the ground-state equilibrium geometry of c-DCE to the ground state of the $4+, 5+$, and $7+$ cations, along with the evolving atomic charges, atomic speeds, and WBIs. Figure S4 also provides comparative data for the $4+$ cation in its lowest singlet electronic state calculated using methods IIa and I. Again, the two methods predict the same fragmentation products, but the method IIa calculations predict higher product vibrational excitation. Data for the $6+$ cation (calculated by method IIa) are shown in the central panel of Fig. S10. The singlet ground state of the $4+$ cation is predicted to dissociate into two $\mathrm{HCCl}^{2+}$ fragments, which survive to $t>50 \mathrm{fs}$. The $\mathrm{CE}$ of the (quartet) ground state of the $5+$ cation is predicted to show parallels: the extra charge is accommodated by prompt expulsion of an $\mathrm{H}^{+}$ion, but the remaining $\mathrm{HCCl}^{2+}$ and $\mathrm{CCl}^{2+}$ fragments again survive to $t>50 \mathrm{fs}$. Adding more charge results in faster, more extensive fragmentation: the method IIIa calculations predict that the $\mathrm{CE}$ from the (quartet) ground state of the $7+$ cation involves successive loss of two $\mathrm{H}^{+}$ions and one $\mathrm{Cl}^{2+}$ ion, followed by a $\mathrm{C}^{+}$ion from decay of a transient $\mathrm{CCCl}^{3+}$ species. Figure 3 shows the remaining $\mathrm{CCl}^{2+}$ species undergoing an increasingly large amplitude vibrational motion suggesting that it would dissociate to two singly charged atoms if the trajectory had been run for longer. These calculations suggest that the 7+ state of c-DCE lies at the cusp between our definitions of low and high parent ion charge states.

As with FC, details of the fragmentation behavior predicted for these low charge states of c-DCE are seen to be sensitive to the exact choice of the starting geometry, the electronic state of the parent ion, and the theoretical method employed-as illustrated with data for the $6+$ cation in Figs. S10 and S11. As shown in Fig. S10, the CE from the quintet ground state of the $6+$ cation formed by vertical excitation from the equilibrium geometry of the ground state returned by method IIa is predicted to result in four singly charged atomic ions and one $\mathrm{CCl}^{2+}$ moiety that fragments on a longer timescaleproducts that (at $t=50 \mathrm{fs}$ ) are intermediate between those predicted by methods IIIa and IIIb (Table III). Distorting the geometry to the classical turning points of the zero-point asymmetric $\mathrm{C}-\mathrm{Cl}$ stretch 
TABLE III. Summary of ionic product species predicted by methods IIla and IIIb following the CE of cis-1,2-dichloroethene from the lowest energy parent cations of differing spin multiplicity $(2 S+1)$ and charge $Z+$, in the range $3 \leq Z \leq 24$, with the vertical ionization energy (IE) calculated using methods IIla and IIIb and analyzed at $t=50$ fs. The ground-state energy in each case is highlighted in bold. For low charge states, methods Illa and Illb predict some differences in product ions (as shown), where the parent ion is identified simply as $\mathrm{M}^{\mathrm{nt}}$. Also shown for the high parent charge states are the number of positive charges $\left(z_{\mathrm{i}}\right)$ on the atomic cations $i\left(z_{\mathrm{H} 1}\right.$ and $z_{\mathrm{H} 2}$ are unity in all cases and thus not listed), their spin multiplicities $\left(2 s+1\right.$, indicated by the left superscript) returned by the method Illb calculations, and $\sum I E\left(i, z_{i}\right)$, the lowest sum of ionization energies for forming the specified atomic ions. The latter values are taken from the NIST Atomic Database, and the threshold energies for the various $z_{i}+\rightarrow\left(z_{i}+1\right)++$ transitions and energies of the various excited state ions are listed in Tables $S 1$ and $S 2$. The predicted product charge states in rows containing no such entry are as for the parent $Z$ and $2 S+1$ state in the immediate preceding row. The missing entry for the product ion energy sum following the CE of the quintet C-DCE ${ }^{2+}$ ion reflects the lack of an excitation energy for the lowest quartet state of the $\mathrm{Cl}^{6+}$ ion.

\begin{tabular}{|c|c|c|c|c|c|c|c|c|}
\hline \multirow[b]{2}{*}{$Z$} & \multirow[b]{2}{*}{$2 S+1$} & \multicolumn{2}{|c|}{$I E / \mathrm{eV}$} & \multicolumn{5}{|c|}{ Product ions predicted by the method } \\
\hline & & IIIa & IIIb & & & & & IIIb \\
\hline \multirow[t]{2}{*}{3} & 2 & 51.6 & 48.1 & & $\mathrm{HC}$ & & & $\mathrm{M}^{3+}$ \\
\hline & 4 & 51.6 & 48.1 & & & & & $\mathrm{M}^{3+}$ \\
\hline \multirow[t]{3}{*}{4} & 1 & 82.1 & 78.4 & & & & & $\mathrm{Cl}^{2+}(\times 2)$ \\
\hline & 3 & 82.1 & 78.5 & & & & & $\mathrm{Cl}^{2+}(\times 2)$ \\
\hline & 5 & 84.2 & 79.5 & & $\mathrm{H}_{2}$ & & & $\mathrm{Cl}^{3+}$ and $\mathrm{Cl}^{+}$ \\
\hline \multirow[t]{2}{*}{5} & 2 & 122.1 & 116.4 & & $\mathrm{H}^{+}(\times 2$ & $\mathrm{Cl}^{2+}$ & & $\mathrm{ll}^{+}$, and $\mathrm{HCCl}^{2+}$ \\
\hline & 4 & 121.5 & 115.8 & & $\mathrm{H}^{+}, \mathrm{C}$ & & & , and $\mathrm{HCCH}^{2+}$ \\
\hline \multirow[t]{3}{*}{6} & 1 & 169.9 & 163.0 & & $\mathrm{H}^{+}(\times$ & & & and $\mathrm{CCl}^{2+}(\times 2)$ \\
\hline & 3 & 169.8 & 163.1 & & $\mathrm{H}^{+}(\times 2$ & $\mathrm{Cl}^{3+}$ & & ${ }^{+}, \mathrm{Cl}^{+}$, and $\mathrm{CCl}^{2+}$ \\
\hline & 5 & 168.8 & 161.9 & & $\mathrm{H}^{+}(\times$ & & & $(\times 2)$, and $\mathrm{Cl}^{+}(\times 2)$ \\
\hline \multirow[t]{3}{*}{7} & 2 & 225.7 & 218.0 & & $\mathrm{H}^{+}(\times 2)$ & $\mathrm{Cl}^{3+}$ & & ${ }^{+}, \mathrm{Cl}^{2+}$, and $\mathrm{C}_{2}{ }^{2+}$ \\
\hline & 4 & 224.9 & 218.4 & & $\mathrm{H}^{+}(\times 2)$ & $\mathrm{Cl}^{3+}$ & & $\mathrm{I}^{2+}$, and $\mathrm{CCCl}^{3+}$ \\
\hline & & & & $z_{\mathrm{C} 1}$ & $z_{\mathrm{Cl} 1}$ & $z_{\mathrm{C} 2}$ & $z_{\mathrm{Cl} 2}$ & $\sum I E\left(i, z_{i}\right)(\mathrm{eV})$ \\
\hline \multirow[t]{3}{*}{8} & 1 & 287.9 & 280.1 & ${ }^{2} 1$ & ${ }^{4} 2$ & ${ }^{2} 1$ & ${ }^{4} 2$ & 123.3 \\
\hline & 3 & 288.2 & 280.3 & ${ }^{2} 1$ & ${ }^{4} 2$ & ${ }^{2} 1$ & ${ }^{2} 2$ & 125.5 \\
\hline & 5 & 288.4 & 280.4 & ${ }^{2} 1$ & ${ }^{4} 2$ & ${ }^{2} 1$ & ${ }^{4} 2$ & 123.3 \\
\hline \multirow[t]{2}{*}{9} & 2 & 359.3 & 351.2 & ${ }^{2} 1$ & ${ }^{2} 2$ & ${ }^{1} 2$ & ${ }^{2} 2$ & 152.2 \\
\hline & 4 & 359.3 & 350.8 & ${ }^{2} 1$ & ${ }^{2} 2$ & ${ }^{1} 2$ & ${ }^{4} 2$ & 150.0 \\
\hline \multirow[t]{3}{*}{10} & 1 & 438.9 & 429.1 & ${ }^{1} 2$ & ${ }^{4} 2$ & ${ }^{1} 2$ & ${ }^{4} 2$ & 172.0 \\
\hline & 3 & 439.2 & 429.4 & ${ }^{1} 2$ & ${ }^{4} 2$ & ${ }^{1} 2$ & ${ }^{2} 2$ & 174.2 \\
\hline & 5 & 439.6 & 429.6 & & & & & \\
\hline \multirow[t]{2}{*}{11} & 2 & 527.8 & 517.2 & ${ }^{1} 2$ & ${ }^{4} 2$ & ${ }^{1} 2$ & ${ }^{3} 3$ & 211.8 \\
\hline & 4 & 527.9 & 517.6 & ${ }^{1} 2$ & ${ }^{4} 2$ & ${ }^{1} 2$ & $1_{3}$ & 213.5 \\
\hline \multirow[t]{3}{*}{12} & 1 & 622.6 & 611.0 & ${ }^{1} 2$ & ${ }^{3} 3$ & ${ }^{1} 2$ & ${ }^{3} 3$ & 251.6 \\
\hline & 3 & 623.1 & 611.5 & ${ }^{1} 2$ & ${ }^{3} 3$ & ${ }^{3} 2$ & ${ }^{3} 3$ & 258.1 \\
\hline & 5 & 625.4 & 613.3 & ${ }^{1} 2$ & ${ }^{3} 3$ & ${ }^{1} 2$ & ${ }^{3} 3$ & 251.6 \\
\hline \multirow[t]{2}{*}{13} & 2 & 731.7 & 717.8 & ${ }^{2} 3$ & ${ }^{3} 3$ & ${ }^{1} 2$ & ${ }^{3} 3$ & 299.5 \\
\hline & 4 & 731.2 & 719.6 & & & & & \\
\hline \multirow[t]{3}{*}{14} & 1 & 844.2 & 831.3 & ${ }^{2} 3$ & ${ }^{3} 3$ & ${ }^{2} 3$ & ${ }^{3} 3$ & 347.4 \\
\hline & 3 & 844.7 & 831.3 & & & & & \\
\hline & 5 & 845.8 & 832.0 & & & & & \\
\hline \multirow[t]{2}{*}{15} & 2 & 969.3 & 955.4 & ${ }^{2} 3$ & ${ }^{3} 3$ & ${ }^{2} 3$ & ${ }^{2} 4$ & 400.7 \\
\hline & 4 & 968.6 & 955.0 & & & & & \\
\hline
\end{tabular}


TABLE III. (Continued.)

\begin{tabular}{|c|c|c|c|c|c|c|c|c|}
\hline \multirow{3}{*}{$\frac{Z}{16}$} & \multirow{3}{*}{$\frac{2 S+1}{1}$} & \multicolumn{2}{|c|}{$I E / \mathrm{eV}$} & \multicolumn{5}{|c|}{ Product ions predicted by the method } \\
\hline & & \multirow{2}{*}{$\begin{array}{c}\text { IIIa } \\
1101.6\end{array}$} & \multirow{2}{*}{$\begin{array}{c}\mathrm{IIIb} \\
\mathbf{1 0 8 7 . 6}\end{array}$} & \multicolumn{3}{|c|}{ IIIa } & \multicolumn{2}{|c|}{ IIIb } \\
\hline & & & & ${ }^{2} 3$ & ${ }^{2} 4$ & ${ }^{2} 3$ & ${ }^{2} 4$ & 453.9 \\
\hline & 3 & 1101.7 & 1087.6 & & & & & \\
\hline & 5 & 1111.7 & 1097.3 & & & & & \\
\hline \multirow[t]{2}{*}{17} & 2 & 1246.1 & 1231.8 & ${ }^{2} 3$ & ${ }^{2} 4$ & ${ }^{1} 4$ & ${ }^{2} 4$ & 518.4 \\
\hline & 4 & 1255.6 & 1241.2 & & & & & \\
\hline \multirow[t]{3}{*}{18} & 1 & 1396.9 & 1382.2 & ${ }^{1} 4$ & ${ }^{2} 4$ & ${ }^{1} 4$ & ${ }^{2} 4$ & 582.9 \\
\hline & 3 & 1408.0 & 1392.6 & & & & & \\
\hline & 5 & 1419.2 & 1403.0 & ${ }^{1} 4$ & ${ }^{2} 4$ & ${ }^{1} 4$ & ${ }^{4} 4$ & 593.5 \\
\hline \multirow[t]{2}{*}{19} & 2 & 1569.5 & 1554.6 & ${ }^{1} 4$ & ${ }^{2} 4$ & ${ }^{1} 4$ & ${ }^{1} 5$ & 650.6 \\
\hline & 4 & 1581.7 & 1565.6 & ${ }^{1} 4$ & ${ }^{4} 4$ & ${ }^{1} 4$ & ${ }^{1} 5$ & 661.2 \\
\hline \multirow[t]{3}{*}{20} & 1 & 1753.1 & 1736.7 & ${ }^{1} 4$ & ${ }^{1} 5$ & ${ }^{1} 4$ & ${ }^{1} 5$ & 718.2 \\
\hline & 3 & 1753.3 & 1736.7 & ${ }^{1} 4$ & ${ }^{3} 5$ & ${ }^{1} 4$ & ${ }^{1} 5$ & 730.4 \\
\hline & 5 & 1768.3 & 1750.8 & ${ }^{1} 4$ & ${ }^{3} 5$ & ${ }^{1} 4$ & ${ }^{3} 5$ & 742.5 \\
\hline \multirow[t]{2}{*}{21} & 2 & 1948.1 & 1931.2 & ${ }^{1} 4$ & ${ }^{1} 5$ & ${ }^{1} 4$ & ${ }^{2} 6$ & 815.2 \\
\hline & 4 & 1949.8 & 1932.6 & ${ }^{1} 4$ & ${ }^{3} 5$ & ${ }^{1} 4$ & ${ }^{2} 6$ & 827.4 \\
\hline \multirow[t]{3}{*}{22} & 1 & 2157.8 & 2141.0 & ${ }^{1} 4$ & ${ }^{2} 6$ & ${ }^{1} 4$ & ${ }^{2} 6$ & 912.1 \\
\hline & 3 & 2159.6 & 2142.6 & & & & & \\
\hline & 5 & 2360.2 & 2342.8 & ${ }^{1} 4$ & ${ }^{4} 6$ & ${ }^{1} 4$ & ${ }^{2} 6$ & \\
\hline \multirow[t]{2}{*}{23} & 2 & 2378.9 & 2361.3 & ${ }^{1} 4$ & ${ }^{2} 6$ & ${ }^{1} 4$ & ${ }^{1} 7$ & 1026.3 \\
\hline & 4 & 2576.9 & 2559.3 & ${ }^{1} 4$ & ${ }^{2} 6$ & ${ }^{1} 4$ & ${ }^{3} 7$ & 1235.8 \\
\hline \multirow[t]{3}{*}{24} & 1 & 2618.7 & 2602.1 & ${ }^{1} 4$ & ${ }^{1} 7$ & ${ }^{1} 4$ & ${ }^{1} 7$ & 1140.5 \\
\hline & 3 & 2812.2 & 2795.2 & ${ }^{1} 4$ & ${ }^{3} 7$ & ${ }^{1} 4$ & ${ }^{1} 7$ & 1350.0 \\
\hline & 5 & 3007.3 & 2990.4 & ${ }^{1} 4$ & ${ }^{3} 7$ & ${ }^{1} 4$ & ${ }^{3} 7$ & 1559.5 \\
\hline
\end{tabular}

vibration is predicted to have minimal effect on the CE dynamics, other than to "steer" the asymmetry of the explosion. The method IIIb data for the 6+ cation shown in Fig. S11 serve to reinforce the earlier observation that in the case of low charge states at least, the predicted CE dynamics may well be sensitive to the choice of the theoretical method and the spin state of the parent cation. In these method IIIb calculations, vertical excitation to the quintet ground state is predicted to result in a prompt, symmetric explosion to six singly charged atomic ions, but equivalent excitations to the closelying excited triplet and singlet spin states of the same $6+$ cation yield, respectively, one and two bound $\mathrm{CCl}^{2+}$ moieties that persist to beyond the calculation end time.

Data for the $7+$ cation of c-DCE are also used to illustrate a feature of the NBO calculation, which affects the time-dependent $\mathrm{NBO}$ and WBI values reported in this paper. Even in the MP2 calculations, the Gaussian default setting calculates the NBO charges from the HF densities, and this has been adopted for the method I, IIa, and most of the IIIa calculations reported here. However, some method IIIa test calculations were also run using MP2 calculated densities. As shown in Fig. S12, the default setting yields smoothly evolving NBO charges and WBI indices. The MP2-derived charges show the same overall trends but also display sporadic unphysical "spikes." These are erroneous and only observed in the post-SCF calculations.

\section{Summary}

The presence of molecular and atomic products in the $\mathrm{CE}$ of both FC and c-DCE molecules prepared in "low" charge states indicates that chemical binding forces influence the fragmentation dynamics and, thus, that the ultimate dissociation of parent ions promoted to charge states with low $Z$ can be far removed from that predicted assuming a "pure" CE regime. None of the fragmentation dynamics calculations reported here are intended to be more than indicative. Rigorous electronic structure calculations that correctly balance the chemical binding and Coulombic repulsive forces even in these simple systems remain a major challenge. Nonetheless, the present calculations provide many useful pointers. The sensitivity of the fragment ion trajectories to competition between chemical binding forces and the Coulombic repulsion has the result that the outcome of the $\mathrm{CE}$ will depend on many properties of the parent cation. 

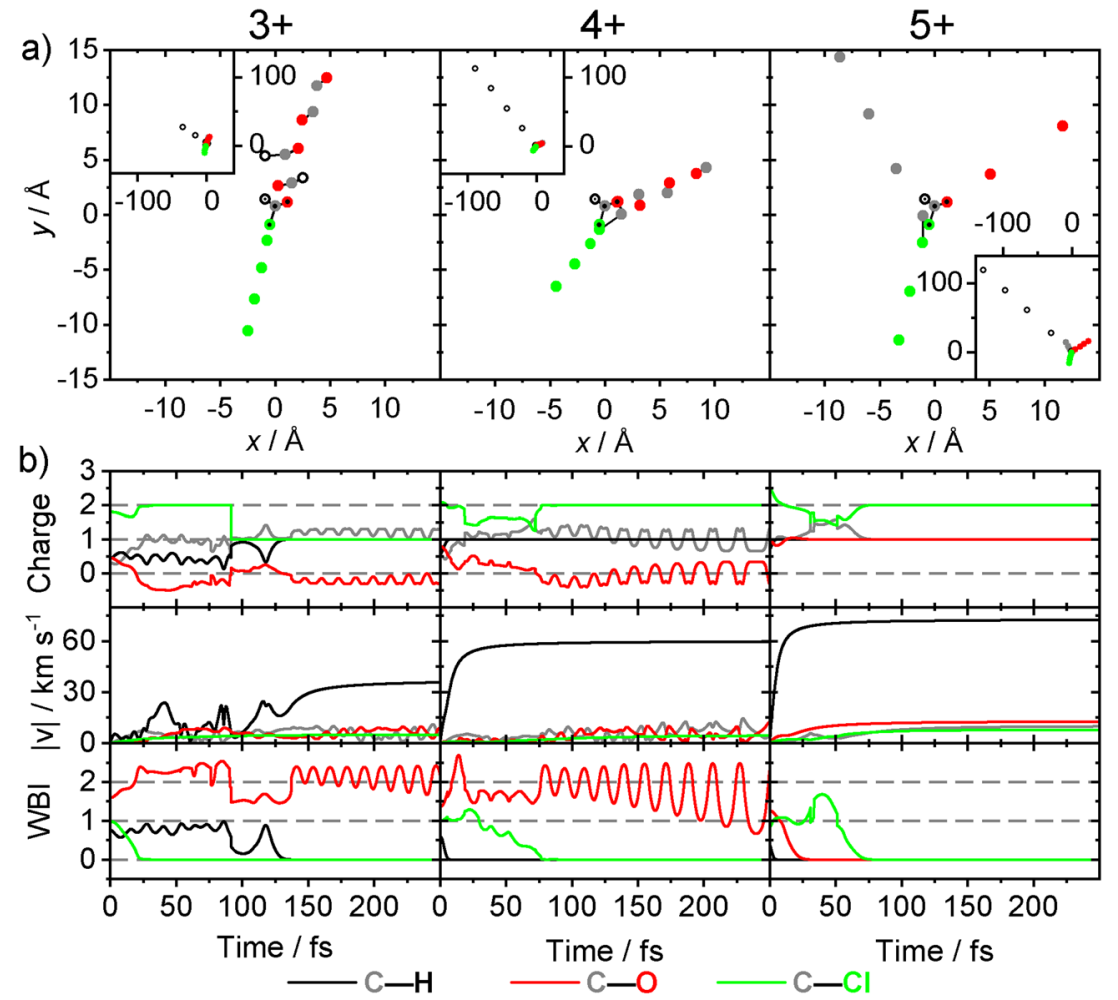

FIG. 2. (a) Fragment trajectories following vertical excitation from the ground-state equilibrium geometry of formyl chloride to the ground electronic states of the $3+, 4+$, and $5+$ cations and (b) time evolving atomic charges, atomic speeds, and WBls calculated by method lla. $\mathrm{H}, \mathrm{C}, \mathrm{Cl}$, and $\mathrm{O}$ atoms are depicted in black/white, gray, green, and red, respectively, with black, green, and red also used for the respective bonds to the $C$ atom, here and in all other plots related to formyl chloride. The point $(0,0)$ in $(a)$ defines the center of mass, the initial nuclear positions are marked with central black dots, the atomic positions are displayed at $\Delta t=62.5 \mathrm{fs}$ intervals (out to $t=250 \mathrm{fs}$ ), and the corresponding positions of the $\mathrm{H}^{+}$nucleus in the respective panels are displayed in the insets on a larger length scale.

These include the following: the molecular geometry immediately prior to ionization, including distortions arising from the zero-point vibrational motion; the total charge on the ion and (see Sec. III B) how rapidly it builds up; the electronic state(s) in which the ion is formed; the non-adiabatic coupling between such states during the CE process; and, in the case of sequential dissociations, the possible Coulomb-induced rotation and/or vibration of the transient intermediates-as demonstrated in recent analyses of the sequential three-body dissociation of $\mathrm{ArCO}^{3+}$ and $\left(\mathrm{CO}_{2}\right)^{3+}$ cations. ${ }^{112}$ Thus, the fragment ion trajectories following the $\mathrm{CE}$ of cations in low $Z$ charge states should rarely be expected to be reliable indicators of the geometry/symmetry of the parent molecule immediately prior to explosion.

Extracting meaningful structural information from images of fragments arising via the CE of "low" charge states experimentally is likely to be equally challenging. Clearly, any such study would rarely produce just one parent ion charge state and would also be likely to access multiple different electronic states for any given $Z+$ cation. Given the foregoing analyses of the CE of low charge states of FC, for example, experiments could be expected to show signals attributable to $\mathrm{H}^{+} \mathrm{C}^{+}, \mathrm{O}^{+}, \mathrm{Cl}^{+}, \mathrm{Cl}^{2+}, \mathrm{CO}^{+}$, and $\mathrm{OCCl}^{+}$ions and more. The relative intensities of these signals will depend not just on the laser pulse intensity (the temporal and spatial distributions of the electric field strengths) but also on any spontaneous or laser-induced secondary dissociation of primary molecular fragments-the products of which need not all be charged. Thus, though there is undoubtedly potential for a multitude of interesting studies aimed at exploring the interplay between the various forces acting within the dissociating polycation, the mapping between initial atomic positions and final fragment velocities during the CE of "low" charge states is very unlikely to be straightforward and the formation and subsequent dissociation of such ions is thus unlikely to be a useful route to probing the neutral parent molecular structure.

\section{B. High parent ion charge states}

As noted above, "high" parent ion charge states are here defined as those that Coulomb explode to yield exclusively atomic cations. Table II lists the atomic charges $z_{i}^{+}$predicted (by method IIa calculations) for the CE products following vertical excitation of neutral FC from its ground-state equilibrium geometry to the ground states (and first excited states of different spin multiplicity) of parent ions with $Z=6-18$. Table II also shows the calculated (method IIa) vertical excitation energies of the various $Z+$ ions and the sum of the ionization energies (IEs) for forming the atomic products in their respective $z_{i}^{+}$charge states. ${ }^{113}$ Table III shows similar data 

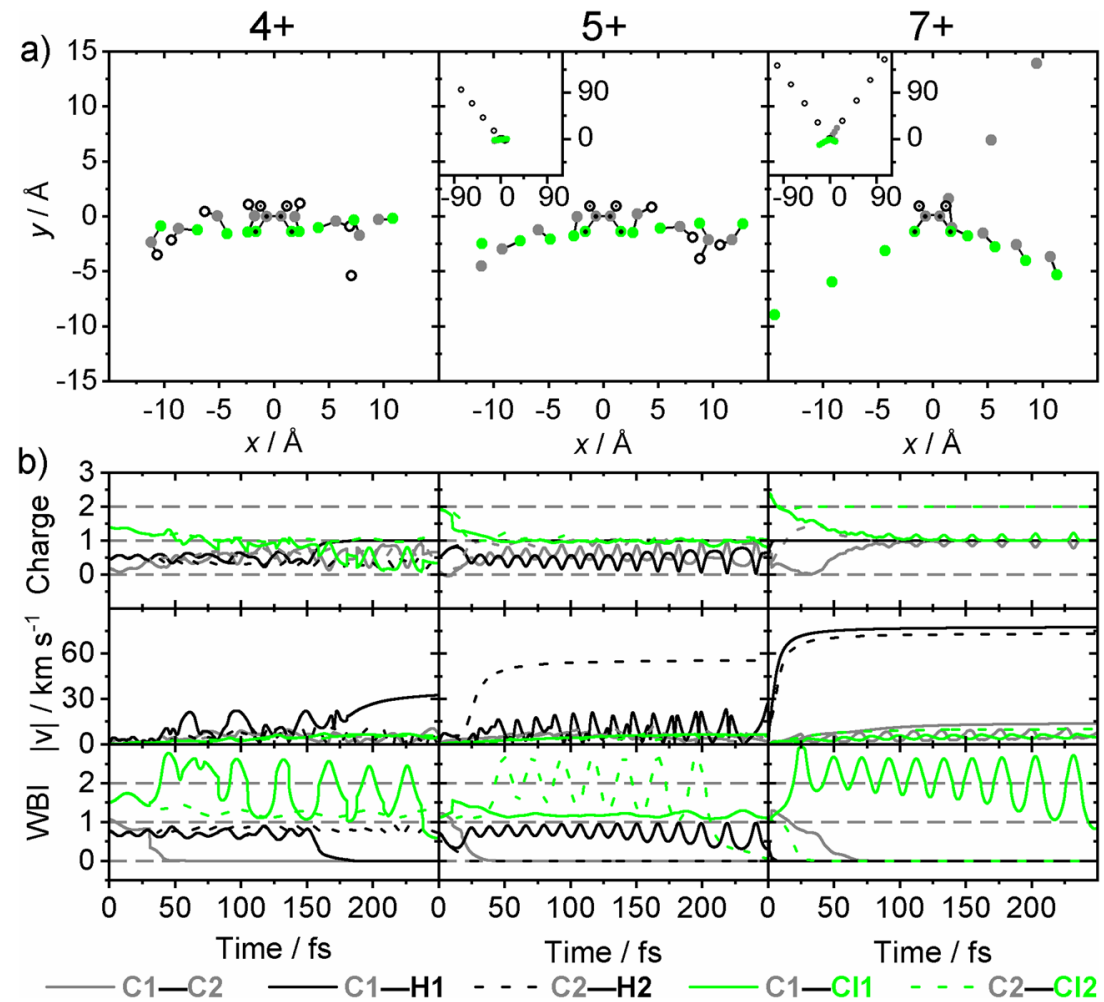

FIG. 3. Fragment trajectories following vertical excitation from the ground-state equilibrium geometry of cis-1,2-dichloroethene to the ground electronic states of the 4+, 5+, and $7+$ cations and (b) time evolving atomic charges, atomic speeds, and WBIs calculated by method Illa. Here and in all other plots involving cis-dichloroethene, the $\mathrm{H}$, $\mathrm{C}$, and $\mathrm{Cl}$ atoms are depicted in black/white, gray, and green, respectively; black, green, and gray are used for the $\mathrm{C}-\mathrm{H}, \mathrm{C}-\mathrm{Cl}$, and $\mathrm{C}-\mathrm{C}$ bonds; the $\mathrm{C}, \mathrm{H}$, and $\mathrm{Cl}$ atoms labeled 1 start with $+x$ (i.e., on the right of the plot); the $\mathrm{C} 2 / \mathrm{H} 2 / \mathrm{Cl} 2$ atoms start at $-x$ (i.e., on the left); and, where applicable, the sum of the $\mathrm{C} 1 / \mathrm{H} 1 / \mathrm{Cl} 1 \mathrm{charges}$ is lower than that of the $\mathrm{C} 2 / \mathrm{H} 2 / \mathrm{Cl} 2$ atoms (i.e., the atoms that have the lower charge sum start on the right). The point $(0,0)$ in (a) defines the center of mass, the initial nuclear positions are marked with central black dots, the atomic positions are displayed at $\Delta t=62.5 \mathrm{fs}$ intervals (out to $t=250 \mathrm{fs}$ ), and the corresponding positions of the $\mathrm{H}^{+}$nuclei in the simulations for the $5+$ and $7+$ cations are displayed in the insets on an expanded length scale. (Note that one $\mathrm{H}^{+}$nucleus detaches in the simulation of the $\mathrm{CE}$ of the $4+$ cation also and travels beyond the lower right corner of the relevant plot by $t=250 \mathrm{fs}$, but not sufficiently far to be clearly visible in an analogous inset box.)

for the CE of different $Z+$ ions of c-DCE, calculated using both methods IIIa and IIIb. These two methods predict slightly different ground-state minimum energy geometries (e.g., the latter predicts a $\sim 2 \%$ longer $\mathrm{C}=\mathrm{C}$ bond length), but both return similar vertical excitation energies and the same fragment ion charges. Clearly, the sum of the atomic charges, $\sum z_{i}$, has to equal $Z$, but, as can be seen by inspecting the various atomic ionization energies listed in Table S1 in the supplementary material, the calculations also reveal an obvious propensity for forming the lowest energy combination of fragment ions, i.e., the combination of products with the minimum $\sum \operatorname{IE}\left(i, z_{i}\right)$. Given the extremely rapid timescale over which the CE occurs, however, the driving force for forming these particular products must be kinetic rather than thermodynamic. We rationalize the preference for forming the lowest energy products by noting that these derive from a "more even" distribution of charge across the fragments. In the pure CE regime, a more even charge distribution results in the strongest overall Coulomb repulsion and thus correlates with the most steeply repulsive Coulomb-determined potential energy surface (PES). The CE will thus occur most rapidly on this PES (relative to any rival PESs), with the result that the thermodynamically favored products are also those that are kinetically favored.

Closer inspection of Tables II and III reveals subtleties, however. For example, the $\mathrm{Cl}^{2+}$ ions from the $\mathrm{CE}$ of the singlet ground state of the $6+$ cation of FC (Table II) are predicted to be formed in their ground quartet state, while those arising as a result of the $\mathrm{CE}$ from the higher-lying triplet and quintet spin states are predicted to be in their low-lying doublet excited state. Similar parent spin-state differences are predicted for the $\mathrm{Cl}^{2+}$ atomic products following the CE of the $8+, 9+$, and $10+$ cations of c-DCE (Table III). Such differences follow naturally if-as in the present modeling-overall spin conservation is required. Table II also suggests that the CE from the ground (quartet spin) state of the $7+$ cation of $\mathrm{FC}$ yields $\mathrm{H}^{+}$and $\mathrm{C}^{2+}, \mathrm{O}^{2+}$ and $\mathrm{Cl}^{2+}$ ions [the last of which in its low-lying excited doublet state (see Table S2 in the supplementary material)], whereas the CE from the lowest energy doublet state of this cation (with a calculated vertical ionization energy just $0.14 \mathrm{eV}$ higher) yields the (slightly) higher energy combination of ground state $\mathrm{H}^{+}, \mathrm{C}^{2+}, \mathrm{O}^{+}$, and $\mathrm{Cl}^{3+}$ ions. Both fragmentations satisfy overall spin conservation, and it is currently unclear whether this difference in predicted 
fragmentation behavior is real or simply a limitation of the applied stability analysis when the energy difference between rival pathways is small. Notwithstanding these subtleties, the differences in predicted product energies are sufficiently small (relative to the overall kinetic energy release upon the CE) that, as shown in Tables II and III, a good first guess of the likely product charge states in the high parent ion charge limit can generally be gained simply by identifying the combination of product ions that gives the smallest $\sum I E\left(i, z_{i}\right)$ sum.

The 16+ through $18+$ cations of FC and the 22+ through 24+ cations of c-DCE each illustrate situations where spin conservation (if applicable) can be expected to have a more dramatic effect on the CE and the fragment ion branching. As shown in Table II, the lowest energy dissociation limit in the case of the $18+$ cation of FC, for example, involves the combination of $\mathrm{H}^{+}, \mathrm{C}^{4+}, \mathrm{O}^{6+}$, and $\mathrm{Cl}^{7+}$, which all have closed shell singlet ground states. Thus, the singlet ground state of this parent ion can explode to these products. However, the lowest triplet excited state of the $18+$ cation is predicted to lie much higher in energy (since its formation requires promotion of a core electron) and to dissociate to a higher energy limit involving $\mathrm{O}^{5+}$ and $\mathrm{Cl}^{8+}$ products, both of which have the doublet ground states necessary in order that the product combination can correlate with a triplet state of the parent. As also shown in Table II, the lowest quintet state of this $18+$ cation constitutes an even more extreme case, in which overall spin conservation dictates that the $\mathrm{Cl}^{8+}$ products are formed in a much higher energy (doubly core excited) quartet excited state. The ground singlet state of the 24+ cation of c-DCE is similarly predicted to dissociate to six closed shell singlet (i.e., two $\mathrm{H}^{+}$, two $\mathrm{C}^{4+}$, and two $\mathrm{Cl}^{7+}$ ) ions. In this case, however, Table III suggests that the lowest energy route to satisfying spin conservation when starting from the much higher-lying triplet and quintet states of the 24+ parent involves formation of either one or both of the $\mathrm{Cl}^{7+}$ ions in their core-excited triplet excited state (Table S2).

Figure 4 shows calculated (method IIa) fragment trajectories for the atomic fragments formed following vertical excitation from the ground-state equilibrium geometry of FC to the ground states of the 9+, 11+, and 14+ cations, along with the evolving atomic speeds and WBIs. Similar data are shown for vertical excitation to the low-lying doublet excited state of the 9+ cation of FC in Fig. S5 (which shows the outputs of method IIa and I calculations to be essentially indistinguishable from one another and from the method IIa predictions for the CE from the quartet ground state shown in Fig. 4). Analogous data for vertical excitation to the ground states of the $11+$ and $14+$ cations of c-DCE are shown in the left and middle columns of Fig. 5 (calculated using method IIIa) and for excitation to the low-lying excited doublet state of the $11+$ cation of c-DCE in Fig. S6. Collectively, these again serve to highlight
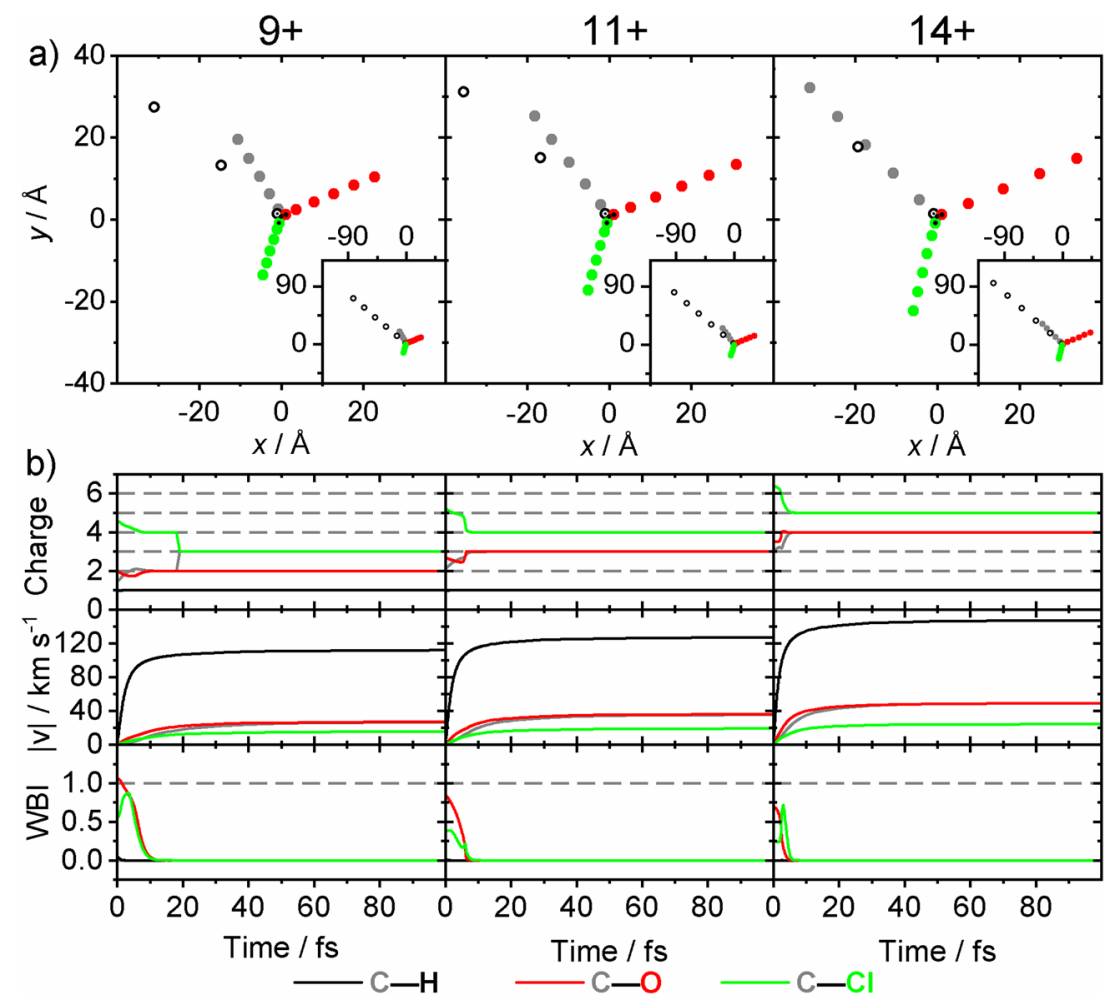

FIG. 4. (a) Fragment trajectories following vertical excitation from the ground-state equilibrium geometry of formyl chloride to the ground electronic states of the $9+$, 11+, and $14+$ cations and (b) time evolving atomic charges, atomic speeds, and WBIs calculated by method lla. The point $(0,0)$ in (a) defines the center of mass, the initial nuclear positions are marked with central black dots, the atomic positions are displayed at $\Delta t=20 \mathrm{fs}$ intervals (out to $t=100 \mathrm{fs}$ ), and the corresponding positions of the $\mathrm{H}^{+}$nucleus in the respective panels are displayed in the insets on a larger length scale. 

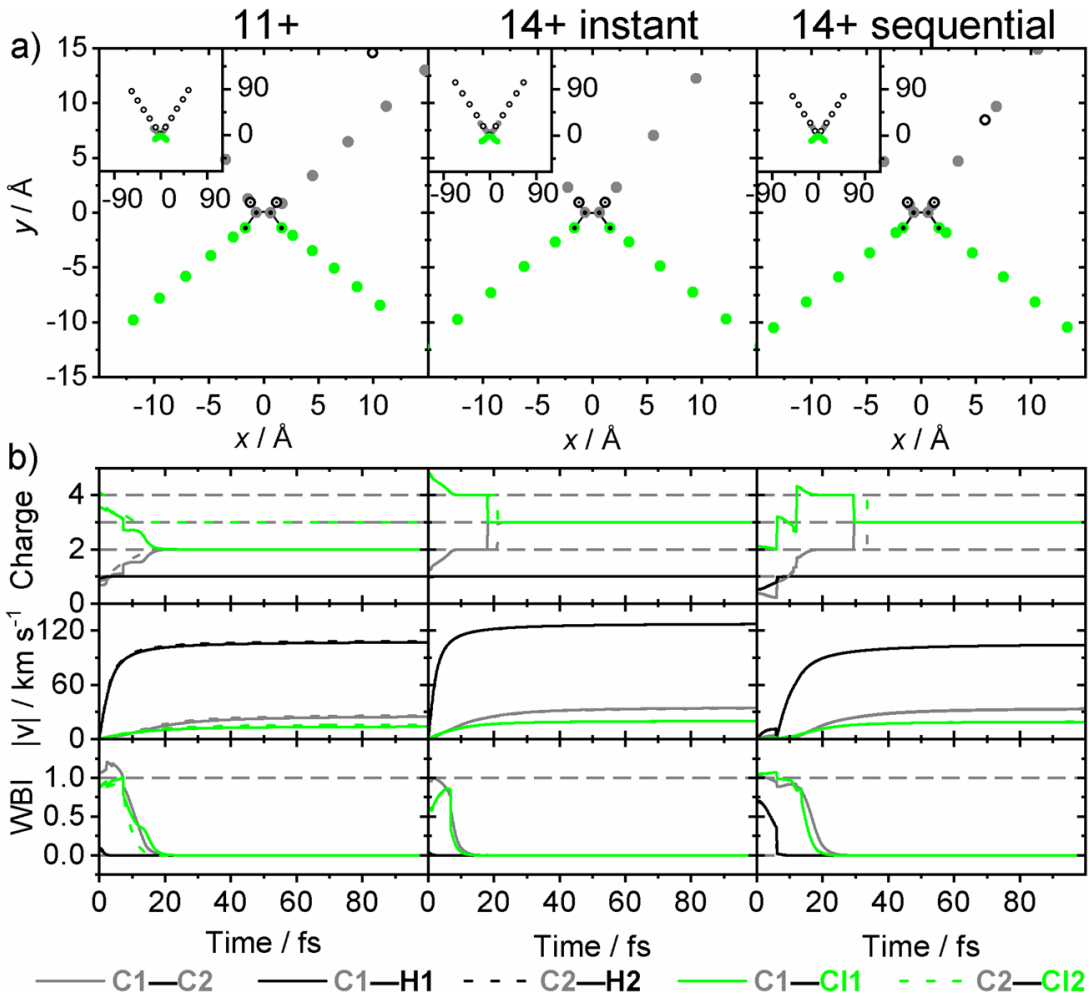

FIG. 5. (a) Fragment trajectories following vertical excitation from the ground-state equilibrium geometry of cis-1,2-dichloroethene to the ground electronic states of the $11+$ and $14+$ cations and (b) time evolving atomic charges, atomic speeds, and WBIs calculated by method IIla. The data shown in the left and center columns assume instantaneous excitation, while those shown in the right column assume sequential excitation via the $4+$ and $8+$ states with a $\delta t=5 \mathrm{fs}$ "waiting time" at each stage. The point $(0,0)$ in (a) defines the center of mass, the initial positions of the various nuclei are marked with central black dots, the atomic positions are displayed at $\Delta t=20 \mathrm{fs}$ intervals (out to $t=100 \mathrm{fs}$ ), the corresponding positions of the $\mathrm{H}^{+}$nuclei in the respective panels are displayed in the insets on a larger length scale, and the 1,2 atom labeling convention is as defined in the caption of Fig. 3.

the very similar outcomes predicted by all three methods for the $11+$ cations. All these figures demonstrate very rapid bond fission (as revealed by the time taken for the various WBIs to drop to zero) and reveal that the charges and the terminal velocities of the atomic fragments from such highly charged parent ions reach their asymptotic values well within $100 \mathrm{fs}$. As expected for systems that are approaching the "pure" CE limit, the fragment ion trajectories are much less sensitive to the variables that affect the trajectories of the charged particles arising from low parent ion charge states. This is illustrated by, for example, comparing the trajectories for the $\mathrm{CE}$ of the quartet ground state of the 14+ cation of FC prepared by vertical excitation from the equilibrium geometry of the neutral ground state or from the inner and outer turning points of the zero-point motion associated with the $v_{2}(\mathrm{C}=\mathrm{O}$ stretch) mode, shown in Fig. S13. The predicted outcomes are essentially indistinguishable.

As noted in the Introduction, some models of the electron dynamics preceding the $\mathrm{CE}$ assume that the parent ion of interest is formed by direct vertical excitation (i.e., the CE "ignites" at the equilibrium geometry of the ground-state neutral molecule), ${ }^{110}$ while others allow for some evolution of the nuclear framework en route to the target $Z+$ state, ${ }^{109}$ which will be reflected in the eventual nuclear dynamics. These different charging scenarios have been investigated by comparing the final fragment ion trajectories when a given $Z+$ charge state is formed either directly or via a sequence of vertical excitations. The sequential model produces intermediate charge states employing a user-defined "waiting time," $\delta t$, with the nuclear geometry permitted to evolve in response to the prevailing chemical bonding and Coulomb repulsion forces. Unsurprisingly, this is yet another variable that can have a substantial influence on the predicted fragment ion trajectories when exploding from a low $Z+$ charge state. However, as shown in Fig. 5, the cDCE $14+$ cation is predicted to display similar, Coulomb-dominated fragmentation dynamics whether prepared directly (middle column) or by sequential excitation via the $Z=4$ and 8 cation states (right column), even when assuming an improbably large value of $5 \mathrm{fs}$ for $\delta t$.

Within the high-charge regime, the respective fragment ion trajectories starting from the ground states of different parent $Z+$ ion charge states are very similar in form, though the terminal speeds $v_{\mathrm{i}}$ of the fragments increase with Z. This is illustrated in Fig. 6(a), which shows the spatial positions of the four fragment ions predicted (method IIa) $100 \mathrm{fs}$ after preparation of ground-state FC cations with $Z$ ranging from 6 to 18 . The $Z$ dependences of the asymptotic speeds of the respective fragment ions are shown in Fig. 6(c). In contrast to the low parent ion charge states, the Coulombic repulsion dominates 


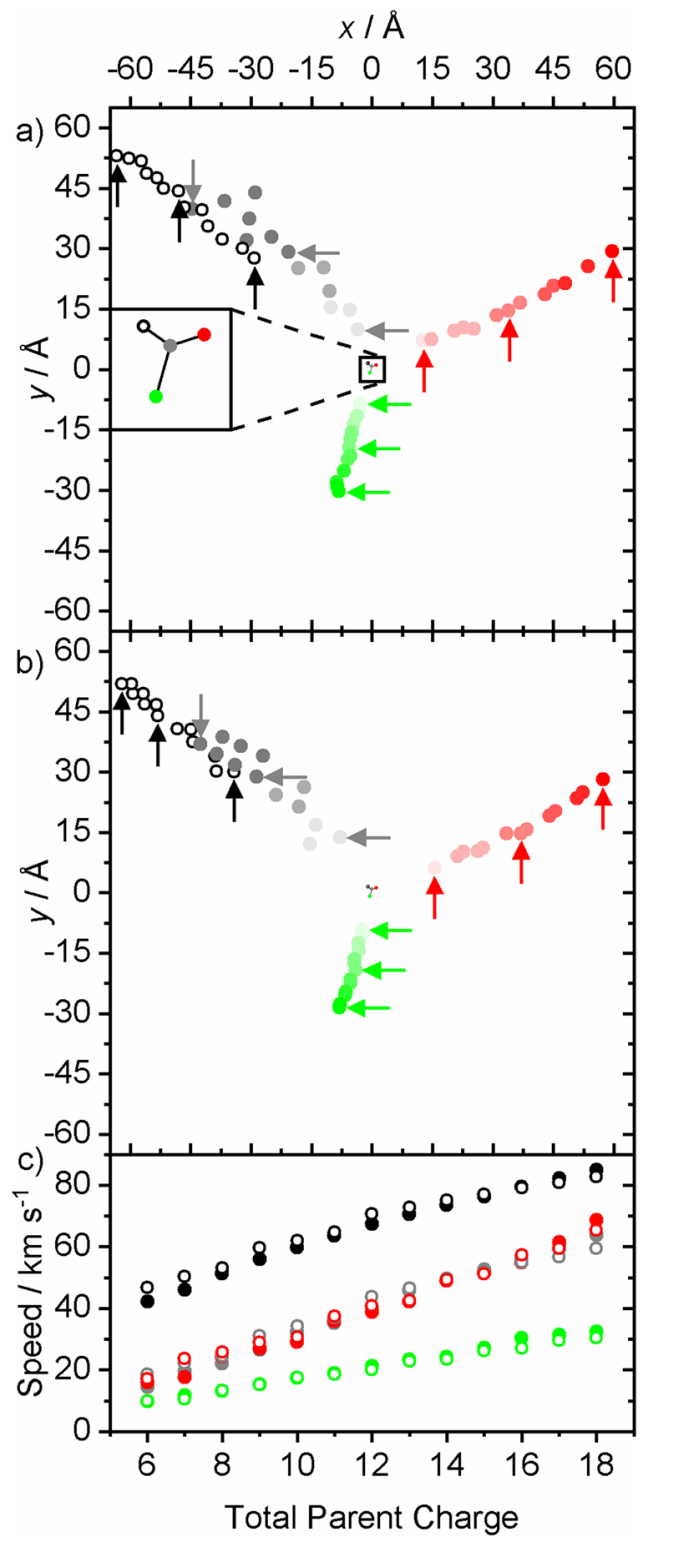

FIG. 6. Relative positions of the fragment ions at $t=100 \mathrm{fs}$ following vertical excitation from the ground-state equilibrium geometry of formyl chloride to the ground-state cations with $Z=6-18$ (displayed using pale through bold points of appropriate color to indicate increasing $Z+$ fragment charges) calculated (a) using method lla and (b) assuming a purely Coulombic model in which the atomic charges at all times are assumed to be the asymptotic charges returned by the method lla calculations. The positions of the $\mathrm{H}^{+}$fragment ions have been divided by 2 prior to inclusion in panels (a) and (b). Panel (c) compares the fragment ion speeds at $t=100 \mathrm{fs}$ predicted by MP2 (filled symbols) and by a pure Coulombic model (open symbols) as functions of $Z$. The arrows in panels (a) and (b) indicate the products of fragmentation when $Z=6,12$, and 18 .

in the high-charge state limit, all bonds are broken, the products are all atomic ions, and, as shown in Fig. 6(b), a purely Coulombic model provides an increasingly adequate description of the fragment ion trajectories. Following the spirit of earlier studies, ${ }^{51}$ the data $x / \AA$

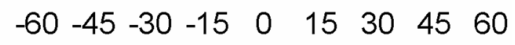

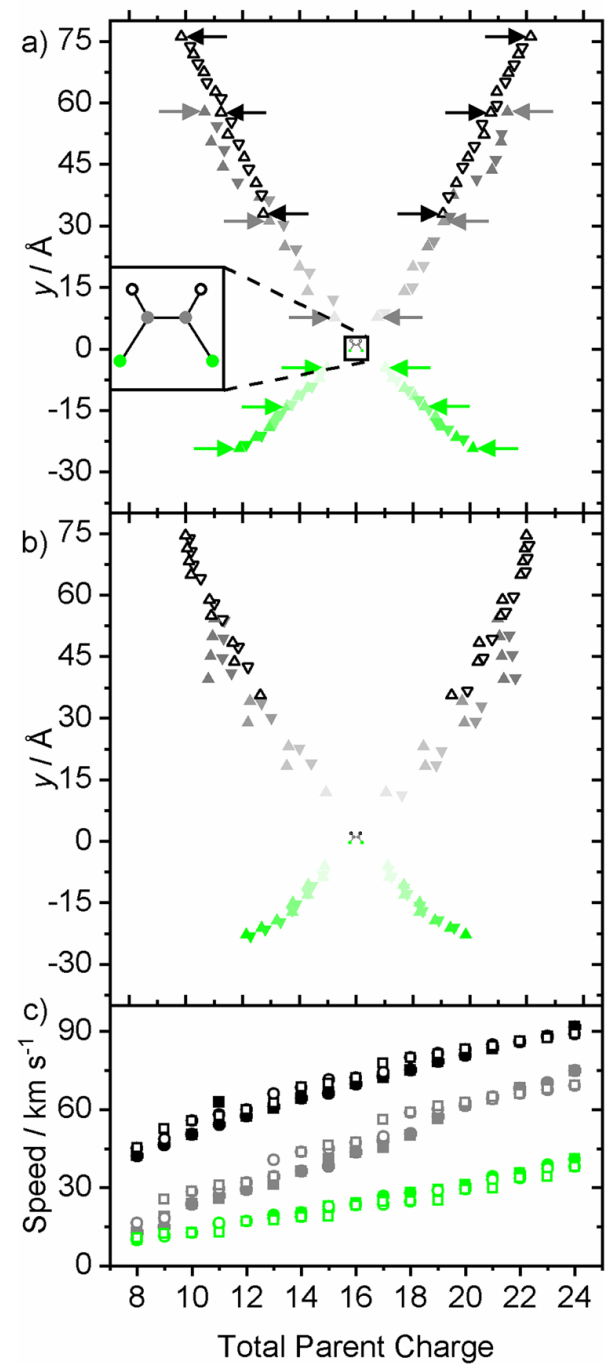

FIG. 7. Relative positions of the fragment ions at $t=100$ fs following vertical excitation from the ground-state equilibrium geometry of cis-1,2-dichloroethene to the ground-state cations with $Z=8-24$ (displayed using pale through bold points of appropriate color to indicate increasing $z+$ fragment charges and upward and downward pointing triangles to distinguish even and odd Z, respectively) calculated (a) using method Ila and (b) assuming a purely Coulombic model in which the atomic charges at all times are assumed to be the asymptotic charges predicted by the method llb calculations. As in Fig. 6, the positions of the $\mathrm{H}^{+}$fragment ions have been divided by 2 prior to inclusion in panels (a) and (b). The arrows in panel (a) indicate the products of fragmentation when $Z=8,16$ and 24 . Panel (c) compares the fragment ion speeds at $t=100$ fs predicted by MP2 (filled symbols) and by a pure Coulombic model (open symbols) as functions of $Z$, using circles to identify atoms on the right side of panel (a) (positive $\mathrm{x}$ ), and squares for atoms on the left.

plotted in Fig. 6(b) have been derived simply by running trajectories starting from the ground-state equilibrium geometry assuming zero initial velocity and the asymptotic charge values for the constituent particles, with the Coulomb repulsion being the only force 

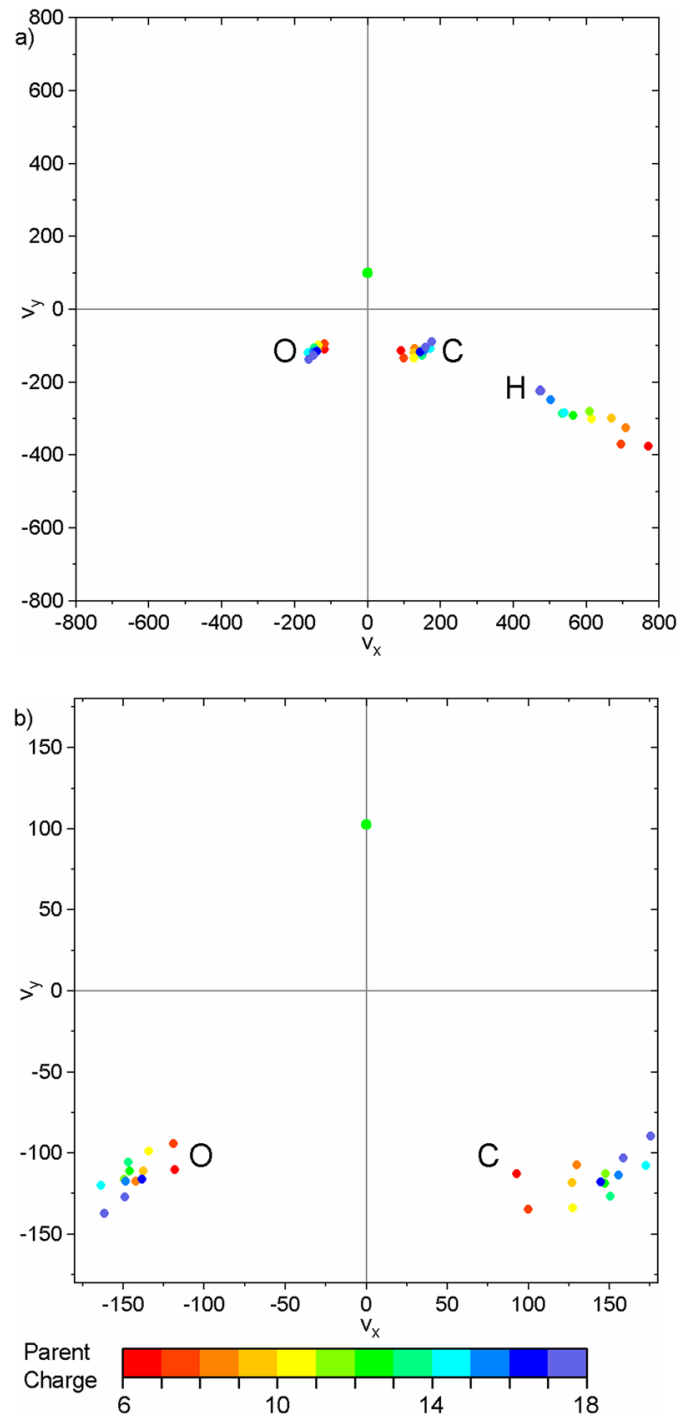

FIG. 8. Plot illustrating the gradual evolution of the scaled relative asymptotic recoil velocities of the fragment ions from the CE of the ground states of different $Z+$ charge states of formyl chloride calculated using method lla. The velocity of each fragment ion has been normalized and rotated so that the marker $\mathrm{Cl}$ ion is at $v_{y}=100, v_{x}=0$, and the different precursor $Z+$ states are color coded. The data in (a) are plotted to include the proton velocities, while (b) shows an expanded view of just the heavy fragment ion relative velocities.

acting on the ions. Figures 7(a) and 7(b) offer a similar comparison of the fragment ion positions predicted by method IIa and by pure Coulombic simulations $100 \mathrm{fs}$ after preparation of ground-state cDCE cations with $Z$ values in the range 8-24. The $Z$ dependences of the asymptotic speeds of the respective fragment ions are compared in Fig. 7(c).

Similarities in the fragment trajectories for different charge states within the high-charge regime can be explored further by comparing scaled versions of the trajectories arising from various initial $Z+$ states. Figure 8 (a) shows a velocity representation of the
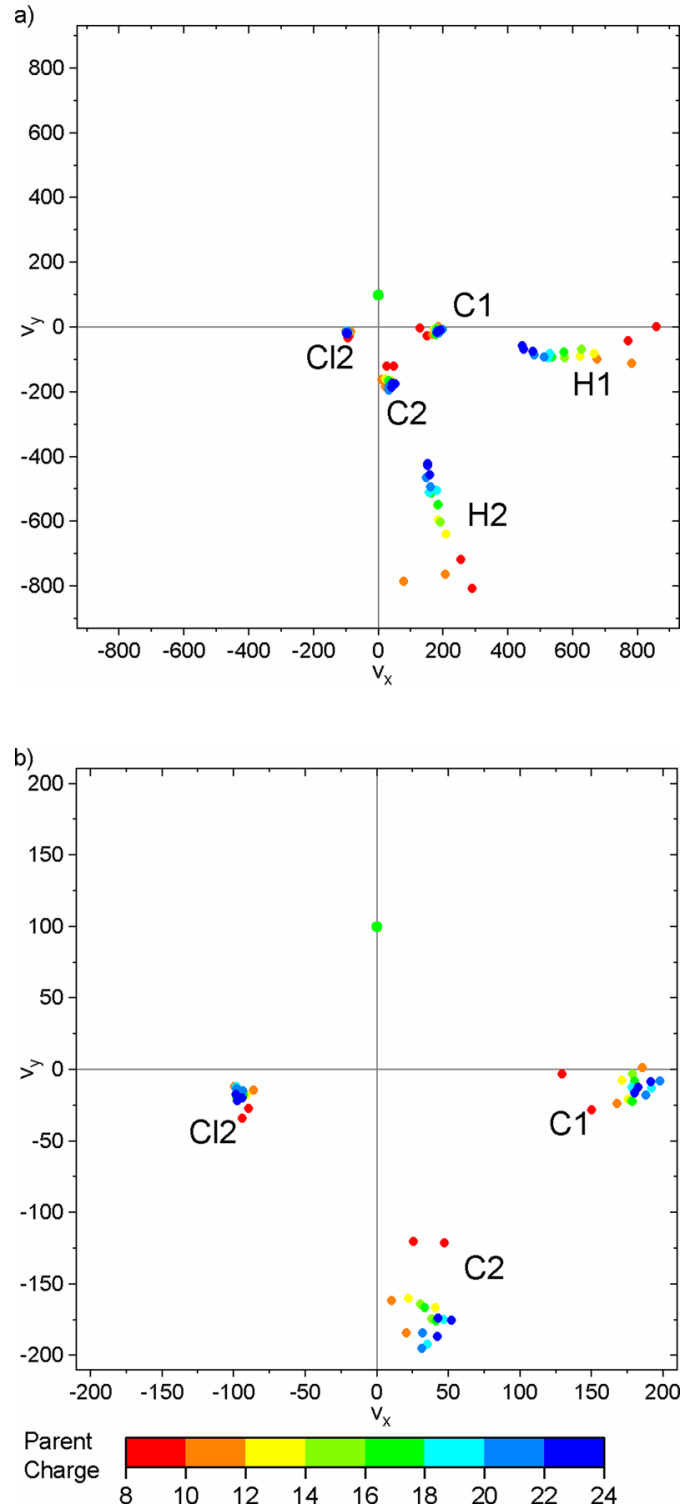

FIG. 9. Plot illustrating the near constancy of the relative asymptotic recoil velocities of the fragment ions from the CE of the ground states of different $Z+$ charge states of cis-1,2-dichloroethene calculated using method lla. The velocities of each fragment ion have been normalized and rotated so that the marker $\mathrm{Cl}$ ion $(\mathrm{Cl} 1)$ is at $v_{Y}=100, v_{x}=0$, and the different precursor $Z+$ states are color coded. The data in (a) are plotted to include the proton velocities, while (b) shows an expanded view of just the heavy fragment ion relative velocities.

FC (method IIa) data presented in Fig. 6 wherein, for each $Z$, the velocities of the fragment ions at $t=100 \mathrm{fs}$ are defined relative to that of one "marker" ion (here chosen to be the $\mathrm{Cl}^{\mathrm{n}+}$ ion). Note that this representation is very similar to that of the experimental datasets obtained via covariance-map imaging analysis, in which the velocities of selected ions are plotted relative to that of a "reference" ion. For each trajectory, the velocities of each fragment ion have been 
scaled and suitably rotated so that the marker atom falls at a common scaled velocity. Figure 8 (b) shows a zoomed plot of the data for just the heavy atomic ions. Similar plots for the CE products from different $Z+$ states of the c-DCE cation are shown in Figs. 9(a) and 9 (b), where all velocities have been scaled relative to that of one $\mathrm{Cl}^{\mathrm{n}+}$ ion (in the case of asymmetric charge distributions, the reference $\mathrm{Cl}^{\mathrm{n}+}$ ion is on the side with the lower total charge).

Viewed in this way, we see that the scaled velocities of the product ions from the CE of parent ions with different (high) $Z$ are similar, falling in clusters on the trajectory plots, but closer inspection reveals several points of note. The scaled velocities of the heavier fragment ions are more similar than those of the light $\mathrm{H}^{+}$ ions. Among the heavy fragment ions, the scaled velocities of those deriving from the $\mathrm{CE}$ of low $Z$ parent ions cluster together least well. The scaled velocities of the heavy ions increase (weakly) with increasing $Z$, whereas the scaled velocities of the $\mathrm{H}^{+}$fragment ions clearly decrease with increasing $Z$. These trends are easily understood, as they follow naturally from the $Z$ dependences of the fragment ion charges shown in Tables II and III. The atomic charges $z_{i}^{+}$for the $i=\mathrm{C}, \mathrm{Cl}$ (and $\mathrm{O}$ ) product ions all increase (individually and/or in total) with increasing $Z$, whereas $z_{\mathrm{H}}{ }^{+}$is capped at one. Thus, the Coulombic repulsion between the light $\mathrm{H}^{+}$ion and the charges on the other (heavier) atoms scales more weakly with $Z$ than the Coulombic repulsions between the heavier fragment ions-consistent with the different $Z$-dependent trends in scaled fragment ion velocities shown in Figs. 8 and 9.

\section{Interpretation of Coulomb explosion velocity-map images in the high-charge regime: Experimental considerations}

The present modeling study encourages the view that the trajectories of the charged atomic ions arising from the CE of sufficiently highly charged parent ion states are useful signifiers of the initial molecular structure. The following discussion focuses solely on the $\mathrm{CE}$ of such highly charged parent ions. CE experiments of the type outlined in Sec. I will normally involve preparation of an aligned distribution of parent ions in a distribution of charge states, so it will rarely be possible to image the dissociation products from a single high $Z+$ parent ion state. However, the present study does suggest a rather consistent mapping from any given $Z+$ parent ion state into the most exoergic combination of $z_{\mathrm{i}}^{+}$fragment ion states. Given such a robust mapping from any given $Z+$ parent ion state into the different $z_{\mathrm{i}}^{+}$fragment ion states, the peak intensities within the recorded fragment TOF spectrum could, in principle, be fitted in order to estimate the $Z+$ charge state distribution of the parent ions. This would allow for some optimization of the $Z+$ distribution by, for example, varying the laser intensity and/or focusing conditions.

The fastest fragments with the highest $z_{\mathrm{i}}^{+}$will derive from the "purest" CE, originating from the most highly charged parent ions. At one level, therefore, these might be considered the fragment ions to target in a CEI experiment designed to determine the parent molecular structure. Against this, however, we recognize that the highest parent charge states considered in this study (wherein the molecule has been stripped of all valence electrons) are probably unattainable experimentally. Furthermore, the resulting fragment ions would have small $\mathrm{m} / \mathrm{z}$ values and fall in the heavily overlapped, early TOF part of the mass spectrum. Imaging any one fragment ion type under such circumstances could be challenging.

However, the data presented in Fig. 8 suggest that similar structural information will be returned by the CE of all high-charge parent ion states. Taking the above practical considerations into account, it might therefore be best to conduct experiments at laser intensities not much above the threshold required to access the highcharge state parent ion regime and to focus on measuring velocity distributions of selected ion charge states identified by the $\mathrm{CE}$ simulations relative to a chosen reference ion in order to extract structural information from the data. In the case of FC, for example, one could consider targeting the $\mathrm{Cl}^{4+}$ fragment ion (Table II) and deriving structural information by using covariance analysis to measure the velocity distributions of the $\mathrm{C}^{3+}$ and the $\mathrm{O}^{2+}$ and/or $\mathrm{O}^{3+}$ fragment ions relative to this ion. Within this analysis, it is entirely possible to eliminate $\mathrm{Cl}^{4+}$ (or any other) ions with velocities below a user-defined threshold in order to focus only on those ions with the highest recoil velocities, generated from parent ions with the highest charges. A very recent work has demonstrated covariance-map imaging in three dimensions, by recording the full $3 \mathrm{D}$ scattering distribution of each fragment rather than just a $2 \mathrm{D}$ projection. ${ }^{40}$ While currently still only at the proof-of-concept stage, this approach offers the promise of being able to measure fragment atomic ion velocities directly in $3 \mathrm{D}$, which will greatly facilitate the reverse mapping from the fragment velocity to the initial atomic position.

\section{CONCLUSIONS}

We report a comprehensive on-the-fly ab initio trajectory study of the Coulomb explosion of gas-phase formyl chloride and cis-1,2dichloroethene that explores the potential use of CE-VMI methods for molecular structure determination. The approach relies on understanding the mapping between the initial atomic positions within the parent molecule and the final velocities of the CE products. For parent ions in low charge states, the present study reveals a complex mapping that is heavily dependent on the initial parent ion charge state and many other experimental and simulation parameters. Several of the gas-phase CE studies reported to date (e.g., Refs. 17, 21, 27, and 36) look to fall within this category; they provide interesting new data about selected aspects of the fragmentation dynamics of parent molecular cations but are generally not suitable for determining the parent structure. For higher parent ion charge states, however, the mapping is shown to become much more straightforward and much less sensitive to the electron dynamics, to the parent charge state, to the electronic state of the ion, or (within reason) to details of the electronic structure or the trajectory propagation methods employed. In this regime, purely Coulombic models are shown to provide a very adequate first-order description of the CE dynamics, and velocity-map images of the resulting atomic ions can be expected to reveal structural information on the parent molecule. CE detection is very well suited for use as the probe step in ultrafast pump-probe experiments and thus holds real promise as a way of mapping time evolving nuclear geometries and thus for studying the early stages of photoinduced fragmentations and ring-opening or cis-trans isomerization processes. As part of the present work, we have considered ways in which trajectory simulations might be used to inform the extraction of quantitative 
information on time-evolving molecular structures from analysis of experimental CE images.

\section{SUPPLEMENTARY MATERIAL}

The supplementary material contains the following: tests of total energy conservation and effects of the trajectory time step size on CE products and product energy disposals using methods I and IIa, and FC and c-DCE 4+ cations, the 9+ cation of FC, and the 11+ cation of c-DCE as exemplars; illustrations of the effects of (i) the zero-point motion in $v_{2}$ (the $\mathrm{C}=\mathrm{O}$ stretch vibration), (ii) the choice of the parent spin state, and (iii) the choice of the electronic structure method on the trajectories of the fragment ions from the CE of the 4+ cation of FC; illustrations of (i) the effects of the parent spin state on the CE dynamics of the $6+$ cation of c-DCE and (ii) the method used to calculate the densities used to derive the timeevolving atomic charges and WBIs (using the CE of the $7+$ cation of c-DCE); illustration of the effects of the zero-point motion in $v_{2}$ (the $\mathrm{C}=\mathrm{O}$ stretch vibration) on the fragment ion trajectories following the CE of the 14+ cation of FC; and ionization and excitation energies of relevant fragment atomic ions.

\section{AUTHORS' CONTRIBUTIONS}

W.Z., G.A.C., and L.G. were responsible for the method I, II, and III calculations, respectively. All authors contributed to the analyses of these data and the preparation of the manuscript. W.Z. and L.G. contributed equally to this work.

\section{ACKNOWLEDGMENTS}

The authors thank the EPSRC for funding via Grant No. EP/L005913/1. M.N.R.A. is very grateful to Dr. Chris Hansen for his many and varied contributions to the early stages of this work, and LG appreciates advice from Dr. Natalie Fey and Professor Fred Manby on the choice of $a b$ initio methods. C.V. and W.Z. would like to acknowledge the use of the Oxford Advanced Research Computing (ARC) facility (http://doi.org/10.5281/zenodo.22558) in carrying out this work.

\section{DATA AVAILABILITY}

The data that support the findings of this study are available within this article and its supplementary material and from the corresponding author upon reasonable request.

\section{REFERENCES}

${ }^{1}$ V. Blanchet, M. Z. Zgierski, T. Seideman, and A. Stolow, Nature 401, 52-54 (1999).

${ }^{2}$ A. Stolow, A. E. Bragg, and D. M. Neumark, Chem. Rev. 104, 1719-1757 (2004).

${ }^{3}$ T. Suzuki, Annu. Rev. Phys. Chem. 57, 555-592 (2006).

${ }^{4}$ P. Hockett, C. Z. Bisgaard, O. J. Clarkin, and A. Stolow, Nat. Phys. 7, 612-615 (2011).

${ }^{5}$ S. Pathak, L. M. Ibele, R. Boll, C. Callegari, A. Demidovich, B. Erk, R. Feifel, R. Forbes, M. Di Fraia, L. Giannessi et al., Nat. Chem. 12, 795-800 (2020).

${ }^{6}$ M. P. Minitti, J. M. Budarz, A. Kirrander, J. S. Robinson, D. Ratner, T. J. Lane, D. Zhu, J. M. Glownia, M. Kozina, H. T. Lemke et al., Phys. Rev. Lett. 114, 255501 (2015).

${ }^{7}$ H. Ihee, V. A. Lobastov, U. M. Gomez, B. M. Goodson, R. Srinivasa, C. V. Ruan, and A. H. Zewail, Science 291, 458-462 (2001).
${ }^{8}$ M. G. Pullen, B. Wolter, A. T. Le, M. Baudisch, M. Hemmer, A. Senftleben, C. D. Schroter, J. Ullrich, R. Moshammer, C. D. Lin et al., Nat. Commun. 6, 7262 (2015).

${ }^{9}$ T. J. A. Wolf, D. M. Sanchez, J. Yang, R. M. Parrish, J. P. F. Nunes, M. Centurion, R. Coffee, J. P. Cryan, M. Gühr, K. Hegazy et al., Nat. Chem. 11, 504-509 (2019).

${ }^{10}$ A. R. Attar, A. Bhattacherjee, C. D. Pemmaraju, K. Schnorr, K. D. Closser, D. Prendergast, and S. R. Leone, Science 356, 54-58 (2017).

${ }^{11}$ P. M. Kraus, M. Zürch, S. K. Cushing, D. M. Neumark, and S. R. Leone, Nat. Rev. Chem. 2, 82-94 (2018).

${ }^{12}$ R. Geneaux, H. J. B. Marroux, A. Guggenmos, D. M. Neumark, and S. R. Leone, Philos. Trans. R. Soc. A 377, 20170463 (2019).

${ }^{13}$ F. Krausz and M. Ivanov, Rev. Mod. Phys. 81, 163-234 (2009).

${ }^{14}$ L. J. Frasinski, K. Codling, and P. A. Hatherly, Science 246, 1029-1031 (1989).

${ }^{15}$ F. Légaré, K. F. Lee, I. V. Litvinyuk, P. W. Doley, A. D. Bandrauk, D. M. Villeneuve, and P. B. Corkum, Phys. Rev. A 72, 052717 (2005).

${ }^{16}$ I. Bocharova, R. Karimi, E. F. Penka, J. P. Brichta, P. Lassonde, X. Q. Fu, J. C. Kieffer, A. D. Bandrauk, I. Litvinyuk, J. Sanderson et al., Phys. Rev. Lett. 107, 063201 (2011).

${ }^{17}$ A. Matsuda, M. Fushitani, E. J. Takahashi, and A. Hishikawa, Phys. Chem. Chem. Phys. 13, 8697-8704 (2011).

${ }^{18}$ M. E. Corrales, G. Gitzinger, J. González-Vázquez, V. Loriot, R. de Nalda, and L. Bañares, J. Phys. Chem. A 116, 2669-2677 (2012).

${ }^{19}$ J. L. Hansen, J. H. Nielsen, C. B. Madsen, A. T. Lindhardt, M. P. Johansson, T. Skrydstrup, L. B. Madsen, and H. Stapelfeldt, J. Chem. Phys. 136, 204310 (2012).

${ }^{20}$ M. Pitzer, M. Kunitski, A. S. Johnson, T. Jahnke, H. Sann, F. Sturm, L. P. H. Schmidt, H. Schmidt-Bocking, R. Dorner, J. Stohner et al., Science 341, 1096-1100 (2013).

${ }^{21}$ H. Ibrahim, B. Wales, S. Beaulieu, B. E. Schmidt, N. Thiré, E. P. Fowe, É. Bisson, C. T. Hebeisen, V. Wanie, M. Giguére et al., Nat. Commun. 5, 4422 (2014).

${ }^{22}$ L. Christensen, J. H. Nielsen, C. B. Brandt, C. B. Madsen, L. B. Madsen, C. S. Slater, A. Lauer, M. Brouard, M. P. Johansson, B. Shepperson, and H. Stapelfeldt, Phys. Rev. Lett. 113, 073005 (2014).

${ }^{23}$ C. S. Slater, S. Blake, M. Brouard, A. Lauer, C. Vallance, J. J. John, R. Turchetta, A. Nomerotski, L. Christensen, J. H. Nielsen et al., Phys. Rev. A 89, 011401 (2014).

${ }^{24}$ C. S. Slater, S. Blake, M. Brouard, A. Lauer, C. Vallance, C. S. Bohun, L. Christensen, J. H. Nielsen, M. P. Johansson, and H. Stapelfeldt, Phys. Rev. A 91, 053424 (2015).

${ }^{25}$ M. Pitzer, G. Kastirke, M. Kunitski, T. Jahnke, T. Bauer, C. Goihl, F. Trinter, C. Schober, K. Henrichs, J. Becht et al., ChemPhysChem 17, 2465-2472 (2016).

${ }^{26}$ J. D. Pickering, K. Amini, M. Brouard, M. Burt, I. J. Bush, L. Christensen, A. Lauer, J. H. Nielsen, C. S. Slater, and H. Stapelfeldt, J. Chem. Phys. 144, 161105 (2016).

${ }^{27}$ U. Ablikim, C. Bomme, H. Xiong, E. Savelyev, R. Obaid, B. Kaderiya, S. Augustin, K. Schnorr, I. Dumitriu, T. Osipov et al., Sci. Rep. 6, 38202 (2016).

${ }^{28}$ M. Burt, R. Boll, J. W. L. Lee, K. Amini, H. Köckert, C. Vallance, A. S. Gentleman, S. R. Mackenzie, S. Bari, C. Bomme et al., Phys. Rev. A 96, 043415 (2017).

${ }^{29}$ K. Amini, R. Boll, A. Lauer, M. Burt, J. W. L. Lee, L. Christensen, F. Braubetae, T. Mullins, F. Savelyev, U. Ablikim et al., J. Chem. Phys. 147, 013933 (2017).

${ }^{30}$ K. Amini, E. Savelyev, F. Brausse, N. Berrah, C. Bomme, M. Brouard, M. Burt, L. Christensen, S. Düsterer, B. Erk et al., Struct. Dyn. 5, 014301 (2018).

${ }^{31}$ T. Yatsuhashi and N. Nakashima, J. Photochem. Photobiol., C 34, 52-84 (2018).

${ }^{32}$ M. Burt, K. Amini, J. W. L. Lee, L. Christiansen, R. R. Johansen, Y. Kobayashi, J. D. Pickering, C. Vallance, M. Brouard, and H. Stapelfeldt, J. Chem. Phys. 148, 091102 (2018).

${ }^{33}$ J. D. Pickering, B. Shepperson, L. Christiansen, and H. Stapelfeldt, J. Chem. Phys. 149, 154306 (2018).

${ }^{34}$ F. Allum, M. Burt, K. Amini, R. Boll, H. Köckert, P. K. Olshin, S. Bari, C. Bomme, F. Brausse, B. de Miranda et al., J. Chem. Phys. 149, 204313 (2018).

${ }^{35}$ M. E. Corrales, J. González-Vázquez, R. de Nalda, and L. Bañares, J. Phys. Chem. Lett. 10, 138-143 (2019). 
${ }^{36} \mathrm{H}$. Wu, Y. X. Xue, J. Q. Wen, H. Wang, L. H. Bai, W. L. He, R. J. Sun, and W. L. Zheng, RSC Adv. 9, 31853 (2019).

${ }^{37}$ C. Schouder, A. S. Chatterley, F. Calvo, L. Christiansen, and H. Stapelfeldt, Struct. Dyn. 6, 044301 (2019).

${ }^{38}$ E. T. Karamatskos, S. Raabe, T. Mullins, A. Trabattoni, P. Stammer, G. Goldsztein, R. R. Johansen, K. Dlugolecki, H. Stapelfeldt, M. J. J. Vrakking et al., Nat. Commun. 10, 3364 (2019).

${ }^{39}$ A. S. Chatterley, M. O. Baatrup, C. A. Schouder, and H. Stapelfeldt, Phys. Chem. Chem. Phys. 22, 3245-3253 (2020).

${ }^{40}$ J. W. L. Lee, H. Köckert, D. Heathcote, D. Popat, R. T. Chapman, G. Karras, P. Majchrzak, E. Springate, and C. Vallance, Commun. Chem. 3, 72 (2020).

${ }^{41}$ Z. Vager, R. Naaman, and E. P. Kanter, Science 244, 426-431 (1989).

${ }^{42}$ E. P. Kanter, Z. Vager, G. Both, and D. Zajfman, J. Chem. Phys. 85, 7487-7488 (1986).

${ }^{43}$ D. Marx and M. Parrinello, Science 271, 179-181 (1996).

${ }^{44}$ L. Knoll, Z. Vager, and D. Marx, Phys. Rev. A 67, 022506 (2003).

${ }^{45}$ Z. Vager, E. P. Kanter, G. Both, P. J. Cooney, A. Faibis, W. Koenig, B. J. Zabransky, and D. Zajfman, Phys. Rev. Lett. 57, 2793-2795 (1986).

${ }^{46}$ A. T. J. B. Eppink and D. H. Parker, Rev. Sci. Instrum. 68, 3477-3484 (1997).

${ }^{47}$ A. Nomerotski, M. Brouard, E. Campbell, A. Clark, J. Crooks, J. Fopma, J. J. John, A. J. Johnsen, C. Slater, R. Turchetta et al., J. Instrum. 5, C07007 (2010).

${ }^{48}$ C. Vallance, M. Brouard, A. Lauer, C. Slater, E. Halford, B. Winter, S. J. King, J. W. L. Lee, D. Pooley, I. Sedgwick et al., Phys. Chem. Chem. Phys. 16, 383-395 (2013).

${ }^{49}$ X. Llopart, R. Ballabriga, M. Campbell, L. Tlustos, and W. Wong, Nucl. Instrum. Methods Phys. Res., Sect. A 581, 485-494 (2007).

${ }^{50}$ I. Last, I. Schek, and J. Jortner, J. Chem. Phys. 107, 6685-6692 (1997).

${ }^{51}$ L. Poth and A. W. Castleman, Jr., J. Phys. Chem. A 102, 4075-4081 (1998).

${ }^{52}$ E. M. Bringa, Nucl. Instrum. Methods Phys. Res., Sect. B 209, 1-8 (2003).

${ }^{53}$ H. Hartung, B. Fricke, T. Morović, W.-D. Sepp, and A. Rosén, Phys. Lett. A 69, 87-89 (1978).

${ }^{54}$ S. Shimizu, V. Zhakhovskii, F. Sato, S. Okihara, S. Sakabe, K. Nishihara, Y. Izawa, T. Yatsuhashi, and N. Nakashima, J. Chem. Phys. 117, 3180-3189 (2002).

${ }^{55}$ S. Shimizu, V. Zhakhovskii, M. Murakami, M. Tanaka, T. Yatsuhashi, S. Okihara, K. Nishihara, S. Sakabe, Y. Izawa, and N. Nakashima, Chem. Phys. Lett. 404, 379-383 (2005).

${ }^{56}$ Z. P. Wang, P. M. Dinh, P. G. Reinhard, and E. Suraud, Laser Phys. 24, 106004 (2014).

${ }^{57}$ W. Zhou, Ph.D. thesis, University of Oxford, 2018.

${ }^{58}$ M. J. Frisch, G. W. Trucks, H. B. Schlegel, G. E. Scuseria, M. A. Robb, J. R. Cheeseman, G. Scalmani, V. Barone, B. Mennucci, G. A. Petersson et al., Gaussian 09, Revision D.01, Gaussian, Inc., Wallingford, CT, 2013.

${ }^{59}$ R. Seeger and J. A. Pople, J. Chem. Phys. 66, 3045-3050 (1977).

${ }^{60}$ R. Bauernschmitt and R. Ahlrichs, J. Chem. Phys. 104, 9047-9052 (1996).

${ }^{61}$ U. C. Singh and P. A. Kollman, J. Comput. Chem. 5, 129-145 (1984).

${ }^{62}$ B. H. Besler, K. M. Merz, and P. A. Kollman, J. Comput. Chem. 11, 431-439 (1990).

${ }^{63}$ J. P. Foster and F. Weinhold, J. Am. Chem. Soc. 102, 7211-7218 (1980).

${ }^{64}$ F. L. Hirshfeld, Theor. Chim. Acta 44, 129-138 (1977).

${ }^{65} \mathrm{~K}$. B. Wiberg, Tetrahedron 24, 1083-1096 (1968).

${ }^{66}$ L. F. Shampine and M. W. Reichelt, SIAM J. Sci. Comput. 18(1), 1-22 (1997).

${ }^{67}$ J. R. Dormand and P. J. Prince, J. Comput. Appl. Math. 6(1), 19-26 (1980).

${ }^{68}$ C. C. J. Roothaan, Rev. Mod. Phys. 23, 69-89 (1951).

${ }^{69}$ J. A. Pople and R. K. Nesbet, J. Chem. Phys. 22, 571-572 (1954).

${ }^{70}$ M. J. Frisch, M. Head-Gordon, and J. A. Pople, Chem. Phys. Lett. 166, 275-280 (1990).

${ }^{71}$ M. J. Frisch, M. Head-Gordon, and J. A. Pople, Chem. Phys. Lett. 166, 281-289 (1990).

${ }^{72}$ M. Head-Gordon, J. A. Pople, and M. J. Frisch, Chem. Phys. Lett. 153, 503-506 (1988).
${ }^{73}$ S. Sæbø and J. Almlöf, Chem. Phys. Lett. 154, 83-89 (1989).

${ }^{74} \mathrm{M}$. Head-Gordon and T. Head-Gordon, Chem. Phys. Lett. 220, 122-128 (1994).

${ }^{75}$ J. A. Pople, J. S. Binkley, and R. Seeger, Int. J. Quantum Chem. 10(S10), 1-19 (1976).

${ }^{76}$ J. A. Pople, R. Seeger, and R. Krishnan, Int. J. Quantum Chem. 12(S11), 149-163 (1977).

${ }^{77}$ R. Krishnan and J. A. Pople, Int. J. Quantum Chem. 14, 91-100 (1978).

${ }^{78}$ R. Krishnan, M. J. Frisch, and J. A. Pople, J. Chem. Phys. 72, 4244-4245 (1980).

${ }^{79}$ A. D. Becke, J. Chem. Phys. 98, 5648-5652 (1993).

${ }^{80}$ C. T. Lee, W. T. Yang, and R. G. Parr, Phys. Rev. B 37, 785-789 (1988).

${ }^{81}$ B. Miehlich, A. Savin, H. Stoll, and H. Preuss, Chem. Phys. Lett. 157, 200-206 (1989).

${ }^{82}$ S. H. Vosko, L. Wilk, and M. Nusair, Can. J. Phys. 58, 1200-1211 (1980).

${ }^{83}$ T. Yanai, D. P. Tew, and N. C. Handy, Chem. Phys. Lett. 393, 51-57 (2004).

${ }^{84}$ A. D. Becke, J. Chem. Phys. 107, 8554-8560 (1997).

${ }^{85}$ J.-D. Chai and M. Head-Gordon, J. Chem. Phys. 128, 084106 (2008).

${ }^{86}$ O. A. Vydrov and G. E. Scuseria, J. Chem. Phys. 125, 234109 (2006).

${ }^{87}$ T. Vreven, M. J. Frisch, K. N. Kudin, H. B. Schlegel, and K. Morokuma, Mol. Phys. 104, 701-714 (2005).

${ }^{88}$ O. A. Vydrov and G. E. Scuseria, J. Chem. Phys. 126, 154109 (2007).

${ }^{89}$ A. D. Becke, Phys. Rev. A 38, 3098-3100 (1988).

${ }^{90}$ H. Iikura, T. Tsuneda, T. Yanai, and K. Hirao, J. Chem. Phys. 115, 3540-3544 (2001).

${ }^{91}$ R. Peverati and D. G. Truhlar, J. Phys. Chem. Lett. 2, 2810-2817 (2011).

${ }^{92}$ Y. Zhao and D. G. Truhlar, J. Phys. Chem. A 110, 13126-13130 (2006).

${ }^{93}$ R. Ditchfield, W. J. Hehre, and J. A. Pople, J. Chem. Phys. 54, 724-728 (1971).

${ }^{94}$ W. J. Hehre, R. Ditchfield, and J. A. Pople, J. Chem. Phys. 56, 2257-2261 (1972).

${ }^{95}$ P. C. Hariharan and J. A. Pople, Theor. Chim. Acta 28, 213-222 (1973).

${ }^{96}$ P. C. Hariharan and J. A. Pople, Mol. Phys. 27, 209-214 (1974).

${ }^{97}$ M. M. Francl, W. J. Pietro, and W. J. Hehre, J. Chem. Phys. 77, 3654-3665 (1982).

${ }^{98}$ A. D. McLean, J. Chem. Phys. 72, 5639-5648 (1980).

${ }^{99}$ R. Krishnan, J. S. Binkley, R. Seeger, and J. A. Pople, J. Chem. Phys. 72, 650-654 (1980).

${ }^{100}$ T. Clark, J. Chandrasekhar, G. W. Spitznagel, and P. V. R. Schleyer, J. Comput. Chem. 4, 294-301 (1983).

${ }^{101}$ M. J. Frisch, J. A. Pople, and J. S. Binkley, J. Chem. Phys. 80, 3265-3269 (1984).

${ }^{102}$ T. H. Dunning, Jr., J. Chem. Phys. 90, 1007-1023 (1989).

${ }^{103}$ R. A. Kendall and T. H. Dunning, Jr., J. Chem. Phys. 96, 6796-6806 (1992).

${ }^{104}$ D. E. Woon and T. H. Dunning, Jr., J. Chem. Phys. 98, 1358-1371 (1993).

${ }^{105}$ A. Schäfer, H. Horn, and R. Ahlrichs, J. Chem. Phys. 97, 2571-2577 (1992).

${ }^{106}$ A. Schäfer, C. Huber, and R. Ahlrichs, J. Chem. Phys. 100, 5829-5835 (1994).

${ }^{107}$ F. Weigend and R. Ahlrichs, Phys. Chem. Chem. Phys. 7, 3297-3305 (2005).

${ }^{108}$ F. Weigend, Phys. Chem. Chem. Phys. 8, 1057-1065 (2006).

${ }^{109}$ T. Zuo and A. D. Bandrauk, Phys. Rev. A 52, R2511-R2514 (1995).

${ }^{110}$ C. Rose-Petruck, K. J. Schafer, K. R. Wilson, and C. P. J. Barty, Phys. Rev. A 55, 1182-1190 (1997).

${ }^{111}$ NIST Chemistry WebBook, NIST Standard Reference Database Number 69, edited by P. J. Linstrom and W. G. Mallard (National Institute of Standards and Technology, Gaithersburg MD, 2020); retrieved September 29, 2020.

${ }^{112}$ X. Q. Hu, Y. G. Peng, X. L. Zhu, S. C. Yan, L. Liu, W. T. Feng, D. L. Guo, Y. Gao, S. F. Zhang, D. M. Zhao et al., Phys. Rev. A 101, 012707 (2020).

${ }^{113}$ A. Kramida, Yu. Ralchenko, J. Reader, and NIST ASD Team, 2018, NIST Atomic Spectra Database (ver. 5.6.1), available at https://physics.nist.gov/asd, 29 September 2020, National Institute of Standards and Technology, Gaithersburg, NM. 